\title{
Article
}

\section{The Yellow Active Queue Management Algorithm in ICN Routers Based on the Monitoring of Bandwidth Competition}

\author{
Li Zeng ${ }^{1,2} \mathbb{1}$, Hong $\mathrm{Ni}^{1,2}$ and Rui Han ${ }^{1,2, *}$ \\ 1 National Network New Media Engineering Research Center, Institute of Acoustics, Chinese Academy of Sciences, \\ No. 21 North Fourth Ring Road, Haidian District, Beijing 100190, China; zengl@dsp.ac.cn (L.Z.); \\ nih@dsp.ac.cn (H.N.) \\ 2 School of Electronic, Electrical and Communication Engineering, University of Chinese Academy of Sciences, \\ No. 19A Yuquan Road, Shijingshan District, Beijing 100049, China \\ * Correspondence: hanr@dsp.ac.cn; Tel.: +86-1-381-107-7380
}

check for updates

Citation: Zeng, L.; Ni, H.; Han, R. The Yellow Active Queue Management Algorithm in ICN Routers Based on the Monitoring of Bandwidth Competition. Electronics 2021, 10, 806. https://doi.org/10.3390 /electronics10070806

Academic Editor: Francisco Falcone

Received: 5 March 2021

Accepted: 24 March 2021

Published: 29 March 2021

Publisher's Note: MDPI stays neutral with regard to jurisdictional claims in published maps and institutional affiliations.

Copyright: (c) 2021 by the authors. Licensee MDPI, Basel, Switzerland. This article is an open access article distributed under the terms and conditions of the Creative Commons Attribution (CC BY) license (https:// creativecommons.org/licenses/by/ $4.0 /)$.

\begin{abstract}
Deploying the active queue management (AQM) algorithm on a router is an effective way to avoid packet loss caused by congestion. In an information-centric network (ICN), routers not only play a role of packets forwarding but are also content service providers. Congestion in ICN routers can be further summarized as the competition between the external forwarding traffic and the internal cache response traffic for limited bandwidth resources. This indicates that the traditional AQM needs to be redesigned to adapt to ICN. In this paper, we first demonstrated mathematically that allocating more bandwidth for the upstream forwarding flow could improve the quality of service (QoS) of the whole network. Secondly, we propose a novel AQM algorithm, YELLOW, which predicts the bandwidth competition event and adjusts the input rate of request and the marking probability adaptively. Afterwards, we model YELLOW through the totally asymmetric simple exclusion process (TASEP) and deduce the approximate solution of the existence condition for each stationary phase. Finally, we evaluated the performance of YELLOW by NS-3 simulator, and verified the accuracy of modeling results by Monte Carlo. The simulation results showed that the queue of YELLOW could converge to the expected value, and the significant gains of the router with low packet loss rate, robustness and high throughput.
\end{abstract}

Keywords: ICN; active queue management; congestion control; TASEP; QoS; content router

\section{Introduction}

According the statistics from Cisco, global IP traffic has quadrupled over the past 5 years. From 2017 to 2022, IP traffic will continue to grow at an average annual compound growth rate of $26 \%$ [1]. The existing end-to-end communication mode of TCP/IP has been unable to meet the needs of massive content distribution. The traditional TCP/IP network is facing a series of problems such as scalability, mobility, security. Therefore, a promising future network architecture named information-centric networking (ICN) has attracted researchers' attention, such as Data-Oriented (and Beyond) Network Architecture [2], Content-Centric Network (CCN) [3], Network of Information (NetInf) [4], Publish-Subscribe Internet Routing Paradigm (PSIRP) [5]. Rather than gradually patching on the existing network like Content Delivery Network (CDN) [6] or IPv6 [7], ICN attempts to clean-state update the traditional network. In ICN, ubiquitous cache nodes reduce the user's download latency of content and the drain of network bandwidth, which enables efficient content distribution. Adding a new content store (CS) module with complete ICN protocol stack in each router makes the router become a content provider.

In the early research, the high cost Static Random-Access Memory (SRAM) limited the cache capacity of router to multi hundreds of megabytes (MB) [8]. Nowadays, researchers have carried out extensive studies on how to design content router with cache capacity of multi terabytes (TB) [9-11]. For example, in our previous study, in order to make 
the IP network evolve to ICN smoothly, we designed and implemented a router with huge cache capacity [11] through the latest storage performance development kit (SPDK) technology [12]. Chunk is the basic transmission unit in ICN. Some studies believe that link utilization can be improved by setting the size of chunk up to a few MBs due to the substantial overhead brought from small-sized chunks [13]. Owing to the limitation of the network maximum transmission unit (MTU), the content chunk will be cut into many segments to transmit in the network. Routers along the data transmission path collect and assemble these segments into complete chunks, and verify the integrity and correctness of this chunk. The receiver-driven ICN transport protocol ensures the reliable transmission of data chunks, including request loss and timeout retransmission, and traffic congestion control by adjusting the request sending rate in a receiver end system.

As the limit of the ICN router's cache capacity is broken [9], that is, the percentage of cache entries in the total content catalog becomes larger, the cache-hit ratio of the router will naturally increase. With the increase of concurrent services, the throughput requirement of internal cache service traffic will also increase, which will seize port bandwidth and buffer resources of the router against with the external forwarding traffic. Excessive competition will cause congestion, packet loss and may make the Quality of Service (QoS) of the whole ICN network drop sharply. Once any packet is dropped in one node, the chunk that loss packet belongs to will be unable to cache in all the subsequent nodes on the transmission path with high probability, which reduces the utilization of router memory and link. Although the ICN transport protocol contains fast retransmission mechanism, in the heavy load network environment, the round-trip time (RTT) and the queuing delay in each router are enough to release the assembling space allocated from the router memory for this chunk in advance [11]. Many studies [14,15] believe that caching content as close to the network centric node with high betweenness as possible could achieve a large amount of benefit, such as high cache hit rate, lower traffic on link and cache eviction rate. We refute this viewpoint here, because increasing the cache-hit ratio in such a high throughput node will only aggravate the bandwidth competition.

There are two typical congestion control mechanisms in a traditional TCP/IP network, which are both effective methods to improve QoS. One is based on end to end. However, only relying on the source end to reduce the sending window cannot relieve the buffer overflow caused by burst flow effectively, which is the design defect of loss-based transmission protocol [16]. Since the router is the closest congestion perceiver in the network, various network metrics contribute to measure the congestion in real time. Therefore, the other is deploying an active queue management (AQM) algorithm on router, which discards packets adaptively. Afterwards, congestion information will be implicitly or explicitly notified (ECN) to the source for further adjustment, which improves the transmission efficiency of the network [17]. In ICN, most of the congestion control research is carried out around with end-to-end [18-20], which imitates the window-based congestion control mechanism in TCP. Considering the content characteristics of multi-source and multi-path in ICN, these methods usually maintain multiple timeout values and multiple windows to judge and control congestion, which makes the content router maintain a large number of flow states and increases the complexity of congestion control. There are also some research works $[21,22]$ that judge congestion through the assistance of intermediate router nodes. Like some AQM algorithms in IP networks, these methods judge congestion by queue occupancy or packet input rate. Nevertheless, they do not perform packet loss operation on the router, they just inform the receiver to adjust the sending rate of the request packet. It is difficult for these methods similar to the ECN mechanism in the IP network to avoid global synchronization of traffic. Moreover, owing to the well-known differences between ICN and IP, including receiver-driven flow control, stateless forwarding, symmetric paths, we believe that deploying the traditional AQM algorithm in the ICN router directly and mechanically can not achieve significant performance improvement.

In summary, the contribution of our work comprises: 
- We analyzed how additional end attributes in ICN router contributes to congestion through probability theory. Afterwards, we proposed a novel active queue management algorithm YELLOW based on bandwidth competition prediction and limitation for ICN content routers. YELLOW provides a new idea for the design of ICN congestion control algorithm and cache service module in router.

- In order to analyze the correlation between the input rate of request packets and congestion, we modeled the packets' dynamics of bandwidth competition in the YELLOW algorithm through the classic totally asymmetric simple exclusion process (TASEP) model, and deduced the approximate solution of the existence condition for each stationary phase.

- We performed a large number of comparative experiments with several widespread used AMQ algorithms under the ICN environment simulating by NS-3. Experimental results demonstrated the queue of YELLOW can be stable at the expected value, and the significant gains of a router with low packet loss rate, robustness and high throughput. Moreover, we conducted extensive experiments to verify the accuracy of our analysis model for the YELLOW algorithm.

The remainder of this paper is organized as follows. Section 2 provides related research of our work. Section 3 presents the cause and solution analysis of congestion in ICN and the design of YELLOW. The TASEP model analysis of the traffic behavior pattern in YELLOW is presented in Section 4. Our experimental results are shown in Section 5. Finally, we conclude the paper and describe our future work in Section 6.

\section{Related Work}

\subsection{Active Queue Management}

Since the change of internal state of the network can not be detected accurately in the end system, it is more effective and timely to detect and adjust congestion in a router than the window-based TCP congestion control strategy. The simplest drop-tail algorithm is widely used at the earliest, but the long-term full queue will lead to TCP global synchronization, queue deadlock and other network performance problems. Floyd et al. [23] proposed the random early detection (RED) algorithm in 1993 to overcome the shortcomings of drop-tail, which is the earliest standard AQM scheme recommended by the Internet Engineering Task Force (IETF). RED estimates congestion and calculates the marking probability according to the queue length. In the past 20 years, many AQM algorithms have been proposed, which can be divided into two types: one is the optimization algorithm based on RED, the other is designed based on control theory. The performance of the RED algorithm is closely related to the fluctuation of network traffic and the different settings of parameter configuration. Meanwhile, the lack of fairness of RED is neatly illustrated by the fact that the burst flow will quickly occupy the queue resources, which leads to the abnormal transmission of other flow. Therefore, many improved algorithms are proposed, especially GRED [24], ARED [25], SRED [26] and BLUE [27]. SRED algorithm is so complex that it consumes abundant router memory resources, resulting in low scalability. ARED and GRED make the algorithm adaptive and keep the queue length convergent by dynamically adjusting the packet loss probability, although the problem of parameter sensitivity has not been solved perfectly. Compared with the above algorithms, BLUE achieves the lowest packet loss rate with the lowest memory overhead while including a scheduling algorithm based on the Bloom filter to ensure the fairness of each flow.

Misra [28] et al. first proposed a feedback control model based on TCP to optimize the $A Q M$ algorithm while they gave the exact solution. The adaptive virtual queue algorithm (AVQ) [29] achieves a proper balance between high utilization and low delay through using linear differential equations to adjust the virtual capacity of the router. However, due to the lack of explicit control to queue length, the performance of AVQ is not ideal. Ünal [30] et al. introduced the proportional integral (PI) controller to maintain the stability of the queue, but it has the defects of slow response speed and overdependence on buffer size. With the improvement of artificial neural network and fuzzy logic control theory, some 
researchers tried to apply intelligent control theory to design an AQM algorithm based on a proportion integral differential (PID) controller. The self-learning ability of a neuron can adjust the three parameters of PID online, so that these algorithms [31,32] can obtain stable performance under the fluctuating network environment.

Not only can control theory be used to model the congestion caused by limited bandwidth and buffer resource of content routers, the well-assessed queuing theory is a common method to analyze and improve the performance of resource-constrained networks. For example, to eliminate the coupling effect between multiple flows in the router on the packet forwarding rate, [33] deployed multiple decoupled queues on a single node and calculated the ideal transmission rate of packets through the $\mathrm{M} / \mathrm{D} / 1$ queuing model. Besides, $[34,35]$ utilized queuing theory model called Jackson networks $(M / M / k$ open queueing networks) [36] to analyze the dependence between service response time and node capacity constraints, and deduces the optimal cache allocation to meet the required cost/resource tradeoff.

Nowadays, there are few research works that apply the AQM algorithm on ICN. In an ICN-5G network scenario, to satisfy the strict delay constraint from the perspective of transmission control, Shahin et al. proposed a centralized AQM algorithm including load balancing and rerouting [37]. Moreover, Wang et al. [38]. proposed an AQM algorithm in which congestion is detected by monitoring the queuing time of packets. Although this method could achieve high link utilization, the simulation experiment was limited bandwidth to $100 \mathrm{Mbps}$. We believe that the high complexity of this algorithm may make it difficult for ICN routers to maintain line speed forwarding in actual deployment.

\subsection{Congestion Control in Information-Centric Network (ICN)}

Although ubiquitous cache nodes in ICN reduce redundant transmission traffic on the network and achieve efficient content distribution, congestion may still occur. ICN has yet to determine its approach to congestion control, including whether window-based or rate-based congestion control would be the best. Since the window-based congestion control has been studied and deployed widely in the IP network, the majority of research on ICN congestion control focuses on it as well.

ICP [18] and ICTP [19] detected retransmission timeout (RTO) as congestion signal and adjust congestion window through additive increase multiplicative decrease (AIMD). Since the segments of one content chunk may be scattered around the network, the response delay from different sources varies greatly. Unfortunately, both these two protocols do not consider the influence of multi-source content on RTO value. CCTCP [20] attempted to solve the above problem by maintaining an independent RTO timer for each content source, but the huge cost makes it difficult to extend with a large scale. However, the error of RTO estimation cannot be avoided when the RTT detection is not accurate or the network link is unreliable. M. Amadeo [39] proposed an improved protocol to reduce the error of RTO estimation in the wireless environment of Named Data Networking (NDN) through adjusting the sending gap of interest packets according to the arrival rate of data packets. The "pull" request mode in ICN turn the transmission protocol from the sender-driven to the receiver-driven, which offloads the state management and transmission control to the user. The cache module in the router does not need to save the state of each service flow, which reduces the memory overhead and improves its robustness and performance. Moreover, routers can directly participate in congestion control by shaping the forwarding rate of interest packets, and report congestion information to the requester through NDN acknowledge character (NACK), especially HOBHIS [21] that is a hop by hop congestion control algorithm. [22] considered that the shaping rate should refer to the predicted value of content popularity, which is essentially to reallocate the router bandwidth resources in term of the popularity. However, how to determine and maintain the content popularity for a long time is still an outstanding problem. Karami et al. [40] considered using neural network to predict which link may be congested in the whole network. However, how to design a reasonable neural network model with low training complexity is an urgent problem for this kind of study. The design 
purpose of a loss-based transport protocol is one of the main factor of queue overflow. Therefore, Liu [41] proposed and implemented a delay-based transmission protocol in a wireless environment, which calculates the transmission rate and the maximum transmission window of the request according to the real-time predictive network bandwidth. Experimental results showed that this method improves the flow fairness and robustness, and can effectively reduce the congestion in the network.

\section{The Design of Active Queue Management (AQM) Algorithm for ICN Router}

It is still an unsolved problem whether the traditional active queue management algorithm in a TCP network (such as RED, BLUE) is suitable for ICN or not. Firstly, we analyze how the end system added in ICN routers contribute to congestion. Secondly, we present the main ideas on how to relieve congestion caused by this problem. Eventually, we propose a lightweight active queue management algorithm YELLOW for ICN.

\subsection{Problem Description}

Above all, we attempt to explain the cause of congestion from the perspective of the traffic direction into the router. Figure 1 is a simple architecture diagram, which depicts an ICN content router with two ports. The character $i$ represents the request packets. Suppose a request packet arrives at port 0 at a certain time, the ICN router will check whether the requested content has been cached. If not, the packet will be forwarded from port 1 to the next node according to the flow tables. When the requested data have been stored in the router, chunk will be fetched from cache memory and then put on the ICN protocol stack for transmission. The character $d$ represents the data packets that arrive at port 0 , which will be stored and forwarded by the router at the same time. Without the support of specific network strategy, the higher the cache-hit ratio in router, the higher the query per second (QPS) will be, which means that the throughput of internal service traffic will try its best to encroach on the router bandwidth. The ICN router at the edge of the network may often encounter bursting flow. For example, users watch a new popular movie at the same time. Although ICN can reduce the transmission traffic in the network, it is difficult to avoid congestion and packet loss in the router caused by the bandwidth competition of flow in different directions.

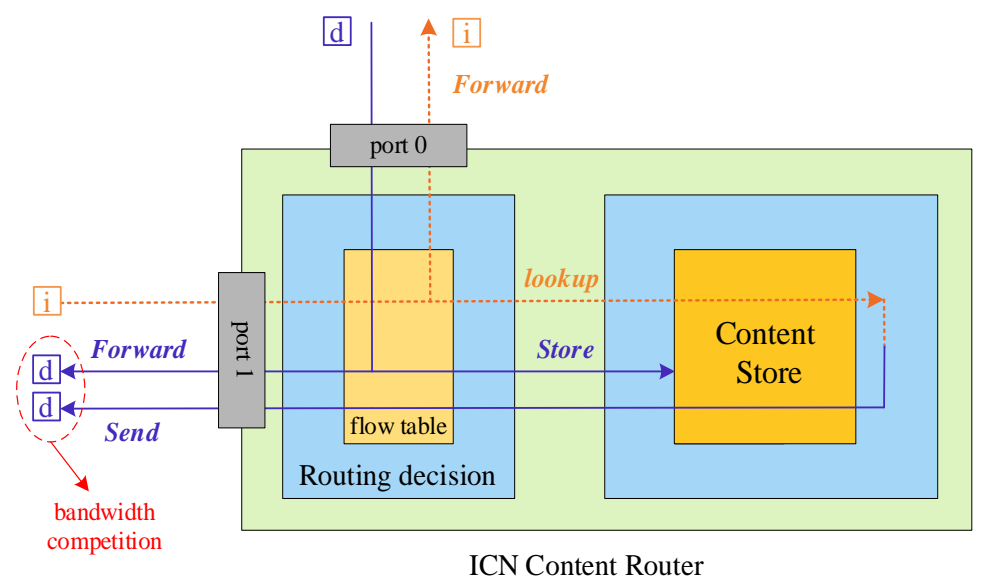

Figure 1. Information-centric network (ICN) router with content store.

Specifically, for port 1 with bandwidth $B_{p}$, bandwidth competition always occurs between external traffic forwarded from port 0 and internal traffic generated from CS in response to request traffic. We believe that the main factor of congestion and packet loss in ICN router cannot be explained by buffer overflow simply, but excessive bandwidth competition at a more practical level. As shown in Figure 2, with the increase of the request arrival rate, the throughput of response traffic $\tau_{c}$ increases while the available bandwidth of forwarding traffic $\tau_{f}$ decreases. At the right side of the figure (gray area), the bandwidth suffers conflict (i.e. $\tau_{f}+\tau_{c}>B_{p}$ ). 


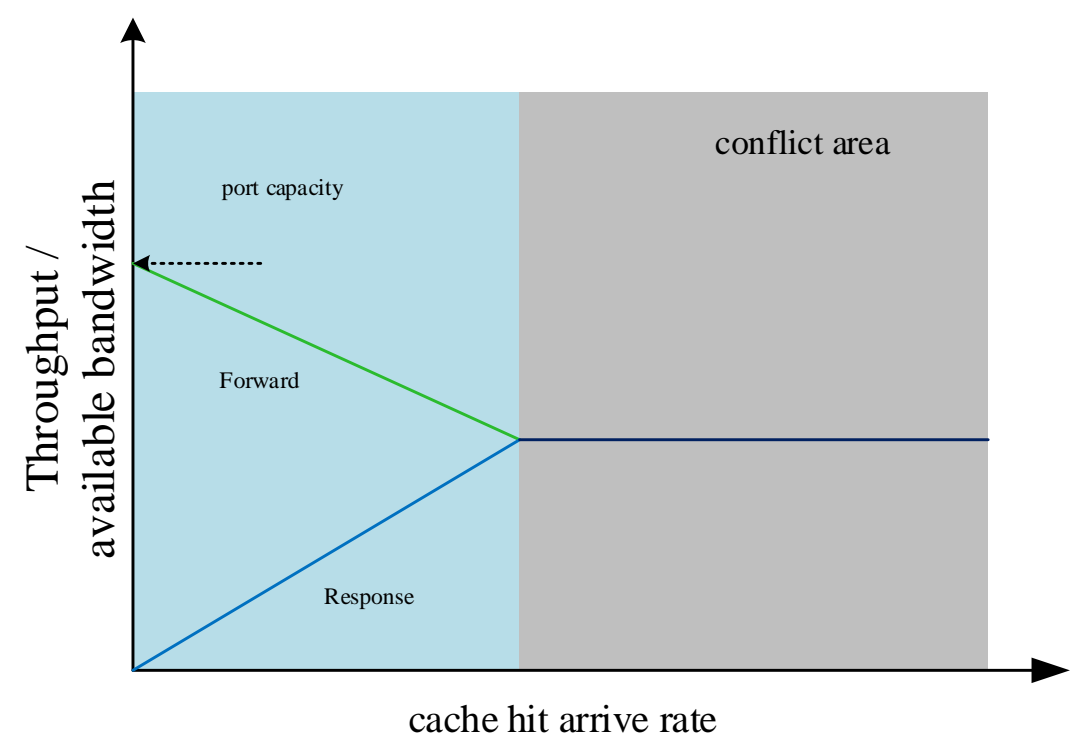

Figure 2. Bandwidth competition.

\subsection{The Priority of Flow in Bandwidth Allocation}

With the increase of router cache capacity, cache hit rate and task concurrency will be improved greatly [11]. Therefore, the real-time bandwidth requirement of cache service traffic cannot be ignored and the conflict may occur at any time. Considering that some classic AQM that drop packets entering the buffer indiscriminately (such as RED and BLUE), we figure that designing an AQM algorithm that can operate differently to the traffics of two directions can improve the QoS of the ICN network more effectively. Now the question is, which direction traffic should have higher priority in bandwidth allocation and how to take care of it carefully when $\tau_{f}+\tau_{c}>B_{p}$. Figure 3 constructs a simple linear network being used to answer the above question from the perspective of probability theory. We allocate port bandwidth by weighting, i.e., $\lambda_{1} \tau_{f}+\lambda_{2} \tau_{c}=B_{p}$, where $\lambda_{1}, \lambda_{2} \leq 1$.

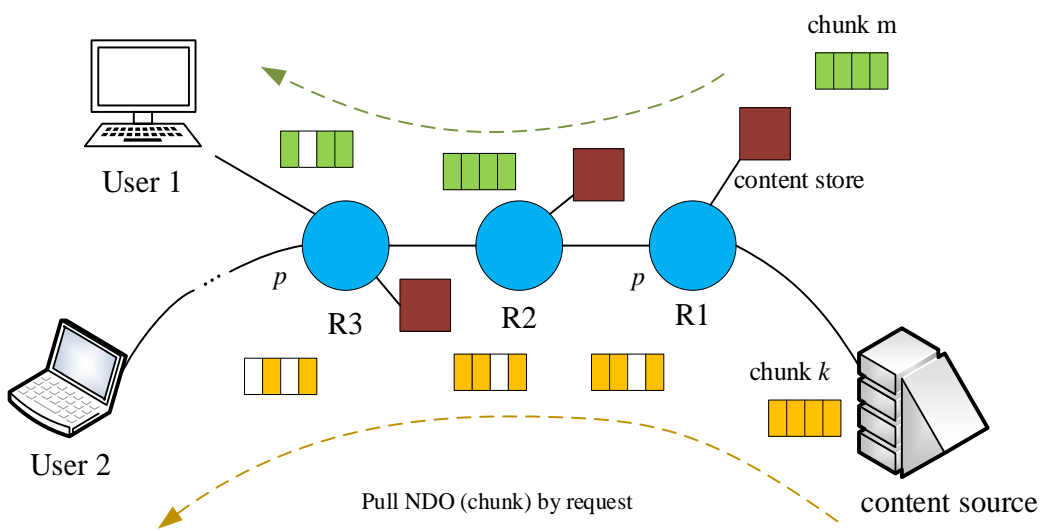

Figure 3. A linear topology using to indicate that forwarding traffic should have higher priority when bandwidth competition happen.

In Figure $3, R_{1}, R_{2}, R_{3}$ are three adjacent ICN content routers with CS (dark red square). $R_{1}$ is the closest to the content source server and the most upstream of the content transmission path. R3 is the closest to the user, so it is located downstream. The data chunk $m$ requested by User1 is located in router $R_{1}$ while User2 request data chunk $k$ from the content source server. Many studies indicate that the large size of chunk can improve the link utilization, such as $4 \mathrm{~K}$ in $\mathrm{CCN}$ [3] or even up to few MBs [13]. Owing to the limitation of the maximum transmission unit (MTU), these large chunks will be segmented into a certain number of packets and be transmitted on the network then. As shown in Figure 3, chunk $k$ and $m$ are 
segmented into 4 packets. The router will collect these packets and then assemble them into a complete chunk for caching in CS. It is supposed that routers adopting traditional AQM will drop the packets indiscriminately with probability $p_{1}$ when congestion occurs. In addition, we analyze two ideal cases of the bandwidth allocation with different priorities under the condition of $\lambda_{1} \tau_{f}+\lambda_{2} \tau_{c}=B_{p}$. One is $\lambda_{1}=1$. In other word, the router tries its best to allocate sufficient bandwidth for external traffic while the cache service traffic is dropped with probability $p_{2}$. The other is $\lambda_{2}=1$, which is the opposite of the above. The router tries its best to ensure the caching service bandwidth while the external forwarding traffic is dropped with probability $p_{2}$. Noted that we can easily deduce that $p_{2}>p_{1}$. Therefore, a single segment of chunk dropped in a router can be regarded as a random independent event and follows the binomial distribution with probability $p_{1} / p_{2}$. We supposed that once there is a packet loss in any node, the chunk that loss packet belongs to will be unable to cache in all the subsequent nodes on the transmission path. The reason is that in a realistic environment, RTT and the queuing delay are enough to release the assembling space allocated from the router memory for this chunk before all the retransmission packets arrive. Tables 1 and 2 summarize the probability and expectation of the number of cache nodes and retransmission for chunk $m$ and $k$, respectively. As the expectation analyses summarized in Tables 1 and 2 show, we can draw a conclusion that the forwarding traffic from upstream deserves more bandwidth, which can bring better benefit to QoS of cache and transmission efficiency when congestion occurs. Note that the linear topology is an important part of complex networks, so this conclusion can be easily extended to any network node.

Table 1. The probability and expectation of the number of cache nodes for chunk $m$ and $k$.

\begin{tabular}{|c|c|c|c|c|c|}
\hline Strategy & Chunk & Cache in 3 Nodes & Cache in 2 Nodes & Cache in 1 Node & Expectation \\
\hline \multirow[b]{2}{*}{ Forwarding without distinction } & $m$ & $\left(1-p_{1}\right)^{8}$ & $\sum^{4} C_{4}^{3} p_{1}^{i}\left(1-p_{1}\right)^{8-i}$ & $\sum^{4} C_{4}^{3} p_{1}^{i}\left(1-p_{1}\right)^{4-i}$ & $\sum^{3} x_{k} p_{k}$ \\
\hline & $k$ & $\left(1-p_{1}\right)^{12}$ & $\sum_{i=1}^{i=1} C_{4}^{3} p_{1}^{i}\left(1-p_{1}\right)^{12-i}$ & $\sum_{i=1}^{i=1} C_{4}^{3} p_{1}^{i}\left(1-p_{1}\right)^{8-i}$ & $\sum_{k=1}^{k=1} x_{k} p_{k}$ \\
\hline \multirow{2}{*}{$\begin{array}{l}\text { Forwarding internal traffic from CS } \\
\text { with high priority } \\
\left(\lambda_{2}=1\right)\end{array}$} & $m$ & $\left(1-p_{2}\right)^{8}$ & $\sum_{i=1}^{4} C_{4}^{3} p_{2}^{i}\left(1-p_{2}\right)^{8-i}$ & te & $\sum_{k=2}^{3} x_{k} p_{k}$ \\
\hline & $k$ & 0 & 0 & 1 & 1 \\
\hline \multirow{2}{*}{$\begin{array}{l}\text { Forwarding external traffic from port } \\
\text { with high priority } \\
\left(\lambda_{1}=1\right)\end{array}$} & $m$ & 0 & 0 & 1 & 1 \\
\hline & $k$ & 1 & 0 & 0 & 3 \\
\hline
\end{tabular}

Table 2. The probability and expectation of retransmission for chunk $m$ and $k$.

\begin{tabular}{ccccc}
\hline Strategy & Chunk & Retransmission & Hop & Expectation \\
\hline & $m$ & $1-\left(1-p_{1}\right)^{12}$ & 3 & $3\left[1-\left(1-p_{1}\right)^{12}\right]$ \\
Forwarding without distinction & $k$ & $1-\left(1-p_{1}\right)^{12}$ & $>3$ & $>3\left[1-\left(1-p_{1}\right)^{12}\right]$ \\
\hline $\begin{array}{c}\text { Forwarding internal traffic from } \\
\text { CS with high priority } \\
\left(\lambda_{2}=1\right)\end{array}$ & $m$ & $1-\left(1-p_{2}\right)^{8}$ & 3 & $3\left[1-\left(1-p_{2}\right)^{8}\right]$ \\
\hline $\begin{array}{c}\text { Forwarding external traffic from } \\
\text { port with high priority } \\
\left(\lambda_{1}=1\right)\end{array}$ & $k$ & 1 & $>3$ & $>3$ \\
\hline
\end{tabular}

\subsection{The Design of YELLOW Algorithm}

In light of the above analysis, we consider that designing an active queue management algorithm for ICN should assign bandwidth with higher priority to the traffic from upstream nodes. Therefore, we propose a novel AQM algorithm called YELLOW, which employs dual queue and real-time requests arrive to monitor congestion caused by bandwidth competition between forwarding and caching. Algorithm 1 shows the details of 
YELLOW, which differs from lots of known AQM algorithms that only use the instantaneous occupancy of a single queue to identify congestion [23-27]. Without increasing the memory overhead, YELLOW sets two queues $Q_{f}$ and $Q_{c}$ in which the forwarding traffic from external and the cache response traffic from internal content stores are queued. Yellow maintains two probabilities $p_{f}$ and $p_{c}$ to drop (mark) packets before they enter the queue. The instantaneous queue length is still a significant monitoring parameter. YELLOW will always firstly increase $p_{c}$ when the packet loss or instantaneous queue length exceed the threshold $\left(L_{1}\right.$ or $\left.L_{2}\right)$, while the change of $p_{f}$ depends on the more restrictions. In ICN, every data packet segmented from one chunk needs to be pulled to user by one request packet [20]. Therefore, the throughput $\tau_{c}$ required by the cache service can be well predicted by the arrival rate of request $\gamma\left(\gamma \leq \tau_{c}\right)$. If $\gamma \geq B-\tau_{f}$ at a certain time, it means that the packet loss rate will rise sharply because of the rapid queue overflow. YELLOW will limit the rate of request packets entering the router by token bucket algorithm, which allows YELLOW to respond quickly to excessive bandwidth competition. Note that by contrast with some AQM algorithms who shape the rate of the request forwarding outward, YELLOW adjusts the rate of the request entering CS. If the explicit congestion notification (ECN) mechanism is allowed, router will set the ECN field in packet header to 1 to inform the requester. On the contrary, if the queue length is lower than the threshold or the link is idle, YELLOW will reduce the marking probability. $\delta_{1}, \delta_{2}, \delta_{3}$ are the values with which the marking probability increases or decreases when an abnormal condition is detected. Moreover, they are set to provide the link ability to effectively adapt to macroscopic changes in load across the link. The value of $\delta_{1}$ should be several orders of magnitude greater than that of $\delta_{2}, \delta_{3}$, which also reflects our biased treatment on cache response traffic and $p_{f}$ should be set to 0 at the beginning. freeze_time is the minimum interval of each update to the marking probability. The value of freeze_time should be set with reference to the effective RTT of the cache service flow in router, so as to allow the changes to be adequately fed back to the requester at the time interval. Over typical links, using freeze_time values between 10 and 200 millisecond (ms) will allow the marking probability to range from 0 to 1 on the order of 5 to $15 \mathrm{~s}$.

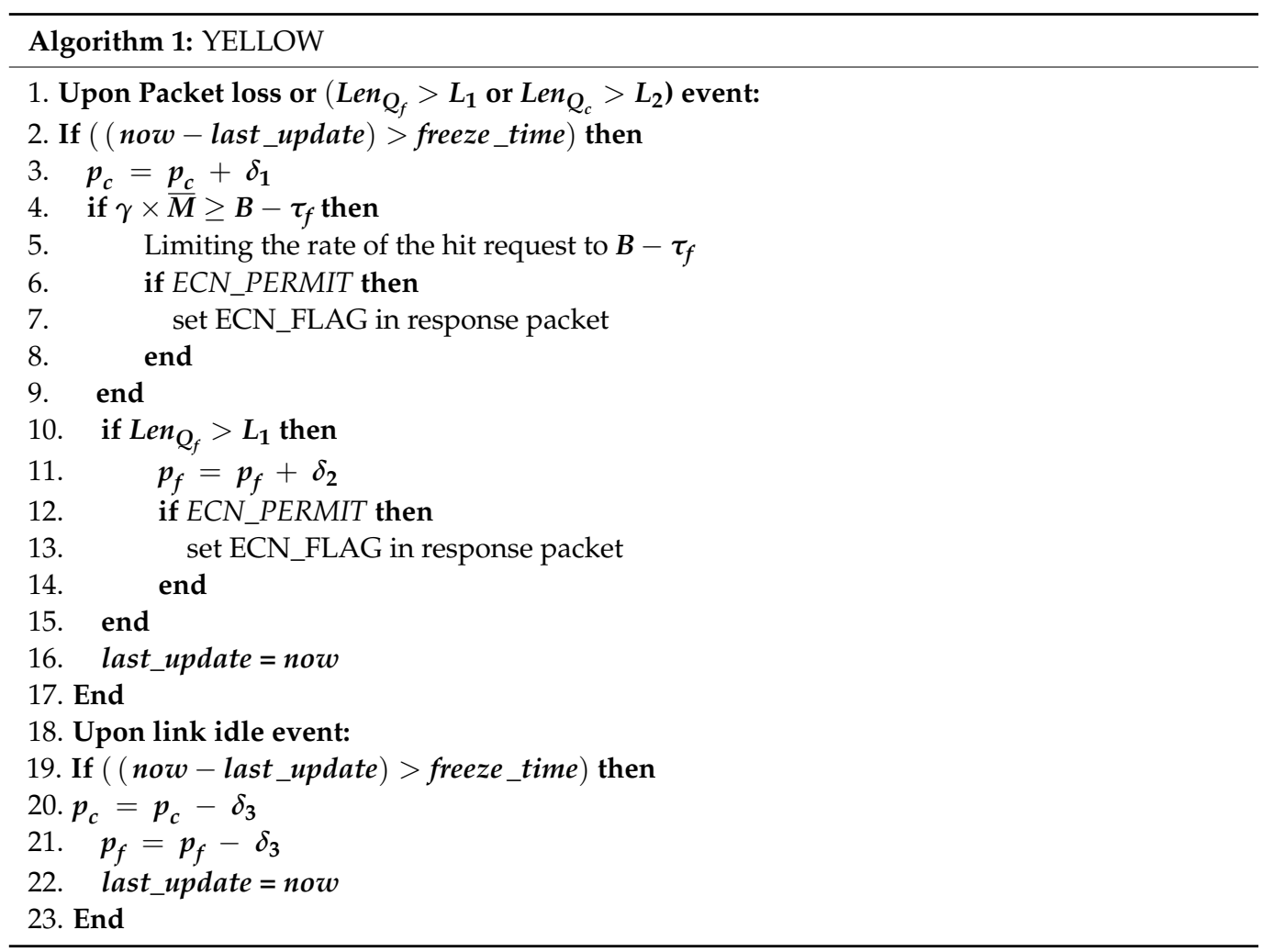




\section{Modeling}

In this chapter, we model the forwarding behavior of packets of the YELLOW algorithm through TASEP [42]. We first introduce TASEP model briefly, and then deduce the existence conditions for each stable phase of YELLOW.

\subsection{Totally Asymmetric Simple Exclusion Process (TASEP)}

TASEP is the most studied model, which is often used to analyze the dynamics and phase transition process of moving objects. The rules of particle motion in the TASEP model are simple, which makes the mathematical analysis easy to deduce. TASEP and its related models can be used to effectively analyze many common statistical physical phenomena, such as simulating road traffic flow and transport process of biological protein molecules. In some relatively simple scenes where the TASEP model is applied, the exact solution can be obtained easily by the Bethe ansatz method [43].

To facilitate understanding, we introduce TASEP through modeling traffic flow on the road. Figure 4 depicts a scene in which many vehicles are driving on a road that can be regarded as a one-dimensional queue with $L$ lattices. As a particle occupying the lattice of the queue, vehicle can only move forward one step when the next lattice in the forward direction is empty. (The model turns to an asymmetric simple exclusion process (ASEP) when a particle in the queue may move forward or backward with different probability at the same time [44].) The vehicle enters the first lattice of the queue with probability $\alpha$ if there is no occupation by any particle. Similarly, the vehicles at the end of the queue jump out of the system with probability $\beta$ at each time step. Each lattice in the system is either empty or occupied by vehicles.

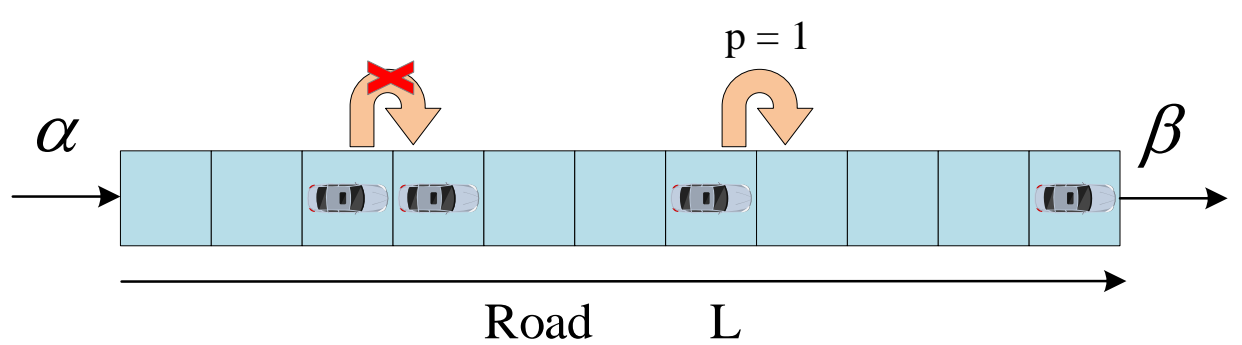

Figure 4. The totally asymmetric simple exclusion process (TASEP) model of traffic flow on road.

System may be in the following three different stationary phases when $\alpha, \beta$ take different values.

1. $\alpha<\beta$ and $\alpha<0$.5. The system is in low-density (LD) phase, which can be regarded as an unblocked road. In LD, the volume of flow J and the particle density $\rho$ of the model are determined by the entering probability $\alpha$. Namely, $\mathrm{J}=\alpha(1-\alpha), \rho_{1}=\alpha$, $\rho_{L}=\alpha(1-\alpha) / \beta, \rho_{\text {bulk }}=\alpha$, where the subscripts 1 , L and bulk denote the head, tail and the middle lattice of TASEP queue, respectively.

2. $\alpha>\beta$ and $\beta<0.5$. The system is in high-density (HD) phase, which can be seen as a congested road. In HD, the volume of flow J and the particle density $\rho$ of the model are determined by the ejecting probability $\beta$. Namely, $J=\beta /(1-\beta)$, $\rho_{1}=1-\beta(1-\beta) / \alpha, \rho_{L}=1-\beta, \rho_{\text {bulk }}=\beta$.

3. $\alpha>0.5$ and $\beta>0.5$. The system is in maximum-current (MC) phase in which the maximum number of vehicles running on the road. And $J=0.25, \rho_{1}=1-\frac{1}{4} \alpha$, $\rho_{L}=\frac{\beta}{4}, \rho_{\text {bulk }}=0.5$.

Moreover, when $\alpha=\beta<0.5$, the system is in a coexistence phase between LD and HD, which is divided by a shock. From this example, we can easily point out that $\alpha$ and $\beta$ can be understood as the normalized relative value between the entrance (exit) rate of the particle and the capacity of intersection in TASEP queue. 


\subsection{Modeling the Dynamics of YELLOW Algorithm through TASEP}

Similarly, we can use TASEP to model the forwarding behavior of packet of YELLOW algorithm in ICN router. Queuing packets in buffer can be regarded as identical particles that move along the lattice. The system consists of three queues with a junction positioned in the middle of the system as shown in Figure 5. Each queue contains $L$ sites. queue 1 and queue 2 merge together at site $2 L+1$. The external forwarding traffic/internal cache service traffic enters queue 1/queue 2 for buffering, and then converges into the port's forwarding queue queue 3 for actual forwarding. Packets enter the queue $1 /$ queue 2 with the rate $\alpha_{1} / \alpha_{2}$ (such as arriving following Poisson distribution). The packets can leave the queue 1/queue $2 /$ system with the rate $\beta_{1} / \beta_{2} / \beta_{3}$. Obviously, in some cases, queue 3 will enter the HD phase because of too much packets entering from queue 1 and queue 2 , which can be used to simulate congestion on the router due to bandwidth conflict. The dynamics of this system modeling is similar to the traffic flow in crossing where two branch roads merge into one primary road. In particular, the primary road is often blocked as well in real life.

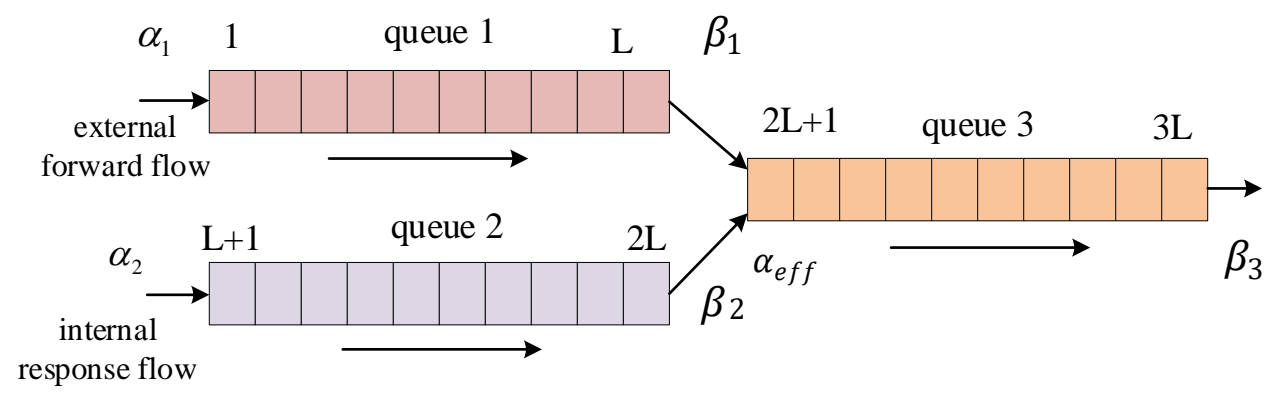

Figure 5. Modeling YELLOW through three TASEP queue with one conjunction.

Since only three stationary phases can be found in each queue, there are possible $3^{3}=27$ stationary phases in our system adopting YELLOW algorithm theoretically. However, the number of possible stationary phases decreases dramatically due to many constraints. For example:

1. Since $\beta_{3}$ can be regarded as the normalization of forwarding capacity of router port, following inequalities are universal: $\alpha_{1}<\beta_{3}, \alpha_{2}<\beta_{3}, \beta_{3}>0.5$. Therefore, queue 3 can only present maximal-current phase or low-density phase. This conclusion can be easily understood, because the purpose of YELLOW algorithm is to avoid keeping the congestion state (HD phase) in the router forwarding queue for a period.

2. According to the principle of flow conservation, the total current passing through the system is equal to:

$$
J_{\text {overall }}=J_{3}=J_{1}+J_{2}
$$

where $J_{1}$ is the volume of flow of queue 1 and $J_{2}$ is that of queue 2 . It suggests that queue 3 cannot have the low-density phase when either queue 1 or queue 2 is in maximal-current phase or High-density phase. Similarly, queue 1 and queue 2 can not be in maximal-current phase at the same time when queue 3 is suffering bandwidth competition (namely at HD phase).

Thus, we infer that there are only six stationary phases in the system: $(L D, L D, L D)$, $(L D, L D, M C),(L D, H D, M C),(L D, M C, M C),(H D, L D, M C)$ and $(M C, L D, M C)$, where $X / Y / Z$ in the expression $(X, Y, Z)$ describes the phase in the queue $1 / 2 / 3$ respectively. Afterwards, we attempt to deduce the approximate solution of existence conditions for these six stationary phases through mean-field theory [45] that neglects the occupation correlations of the sites before and after the junction. Mean-field approximation decomposes many-body problem into multiple one-body problem, which is a common method to study the coupling of multiple TASEP queues. Jonathan $C$ et al. [46] have proved that the power spectrum characteristics of three queues with one junction is the same as that of a single 
TASEP queue. Hence, our system can be analyzed form the perspective of simple coupling of three separate TASEP queues, as the known conclusions introduced in Section 4.1 can be easily utilized such as phase diagram, particle currents and density profiles. In order to facilitate the analysis, we introduce the intermediate variable $\alpha_{e f f}$ as the equivalent entry rate of queue 3.

Firstly, we analyze the simplest stationary phase $(L D, L D, L D)$, which exists when the following inequality is fulfilled:

$$
\alpha_{1}<\frac{1}{2}, \alpha_{1}<\beta_{1}, \alpha_{2}<\frac{1}{2}, \alpha_{2}<\beta_{2}, \alpha_{e f f}<\frac{1}{2}, \alpha_{e f f}<\beta_{3}
$$

According to Equation (1), we can deduce that

$$
\alpha_{1}\left(1-\alpha_{1}\right)+\alpha_{2}\left(1-\alpha_{2}\right)=\alpha_{e f f}\left(1-\alpha_{e f f}\right)
$$

Thus, the possible conditions for the existence of phase $(L D, L D, L D)$ is as follows,

$$
\alpha_{e f f}=\frac{1-\sqrt{1-4\left[\alpha_{1}\left(1-\alpha_{1}\right)+\alpha_{2}\left(1-\alpha_{2}\right)\right]}}{2}<\min \left(\beta_{3}, \frac{1}{2}\right)
$$

For phase $(L D, L D, M C)$, it should satisfy,

$$
\alpha_{1}<\frac{1}{2}, \alpha_{1}<\beta_{1},<\frac{1}{2}, \beta_{2}<\alpha_{2}, \alpha_{e f f}>\frac{1}{2}, \beta_{3}>\frac{1}{2}
$$

Similarly, the possible conditions for the existence of phase $(L D, L D, M C)$ is as follows,

$$
\alpha_{e f f}=\frac{1-\sqrt{1-4\left[\alpha_{1}\left(1-\alpha_{1}\right)+\alpha_{2}\left(1-\alpha_{2}\right)\right]}}{2} \geq \frac{1}{2}
$$

So the only solution of the equation is,

$$
\alpha_{1}\left(1-\alpha_{1}\right)+\alpha_{2}\left(1-\alpha_{2}\right)=\frac{1}{4}
$$

The $(L D, H D, M C)$ phase is defined by the following expression:

$$
\alpha_{1}<\frac{1}{2}, \alpha_{1}<\beta_{1}, \alpha_{2}<\frac{1}{2}, \alpha_{2}<\beta_{2}, \alpha_{e f f}>\frac{1}{2}, \beta_{3}>\frac{1}{2}
$$

Taking into account Equation (1), we obtain:

$$
\begin{aligned}
& \alpha_{1}\left(1-\alpha_{1}\right)+\beta_{2}\left(1-\beta_{2}\right)=\frac{1}{4} \\
& \beta_{2}=\frac{1}{2}-\sqrt{\alpha_{1}\left(1-\alpha_{1}\right)}<\frac{1}{2}
\end{aligned}
$$

At this time, the current on queue $2\left(J_{2}\right)$ should be more than that of queue $1\left(J_{1}\right)$. We could obtain:

$$
J_{1}=\alpha_{1}\left(1-\alpha_{1}\right)<\frac{J_{3}}{2}=\frac{1}{8}
$$

Hence, we can deduce that $\alpha_{1}<\frac{2-\sqrt{2}}{4}$ from (10).

The known conclusion of mean-filed theory for the current [] can be utilized in in our system as follows,

$$
\begin{gathered}
J_{\text {overall }}=\left\langle\tau_{L}\left(1-\tau_{2 L+1}\right)\right\rangle+\left\langle\tau_{2 L}\left(1-\tau_{2 L+1}\right)\right\rangle \\
\approx\left(\left\langle\tau_{L}\right\rangle+\left\langle\tau_{2 L+1}\right\rangle\right)\left(1-\left\langle\tau_{2 L+1}\right\rangle\right) \\
=\left(\rho_{L}+\rho_{2 L}\right)\left(1-\rho_{2 L+1}\right)
\end{gathered}
$$


where $\langle\ldots\rangle$ denotes a statistical average. $\tau_{i}=1\left(\right.$ or $\left.\tau_{i}=0\right)$ indicates that the state of the $i$ th lattice is occupied (or free). Thus the current of each queue can be written as:

$$
J_{1}=\beta_{1} \rho_{L}, J_{2}=\beta_{2} \rho_{2 L}, J_{3}=\alpha_{e f f}\left(1-\rho_{2 L+1}\right)
$$

Therefore, the $\alpha_{\text {eff }}$ can be deduced from (11) and (12) as follows:

$$
\alpha_{e f f}=\rho_{L}+\rho_{2 L}=1-\beta_{2}+\rho_{L}=\sqrt{\alpha_{1}\left(1-\alpha_{1}\right)}-\frac{1}{2}+\rho_{L}>\frac{1}{2}>\alpha_{2}
$$

From (11)-(13), we find the system is in the $(L D, H D, M C)$ phase when:

$$
\alpha_{2}>\frac{1}{2}-\sqrt{\alpha_{1}\left(1-\alpha_{1}\right)}, \alpha_{1}<\frac{2-\sqrt{2}}{4}
$$

Similarly, for phase $(H D, L D, M C)$, it should satisfy,

$$
\beta_{1}<\frac{1}{2}, \beta_{1}<\alpha_{1}, \alpha_{2}<\frac{1}{2}, \alpha_{2}<\beta_{2}, \alpha_{e f f}>\frac{1}{2}, \beta_{3}>\frac{1}{2}
$$

We obtain the following equations according to (1):

$$
\begin{aligned}
& \beta_{1}\left(1-\beta_{1}\right)+\alpha_{2}\left(1-\alpha_{2}\right)=\frac{1}{4} \\
& \beta_{2}=\frac{1}{2}-\sqrt{\alpha_{2}\left(1-\alpha_{2}\right)}<\frac{1}{2}
\end{aligned}
$$

At this time, the current on queue $2\left(J_{2}\right)$ should be less than that of queue $1\left(J_{1}\right)$. We could obtain:

$$
\begin{gathered}
J_{2}=\alpha_{2}\left(1-\alpha_{2}\right)<\frac{J_{3}}{2}=\frac{1}{8} \\
\alpha_{e f f}=\rho_{L}+\rho_{2 L}=1-\beta_{1}+\rho_{2 L}=\sqrt{\alpha_{2}\left(1-\alpha_{2}\right)}+\frac{1}{2}+\rho_{2 L}>\frac{1}{2}>\alpha_{2}
\end{gathered}
$$

Hence, the Equation (15) can be rewritten as:

$$
\alpha_{1}>\frac{1}{2}-\sqrt{\alpha_{2}\left(1-\alpha_{2}\right)}, \alpha_{2}<\frac{2-\sqrt{2}}{4}, \beta>\frac{1}{2}
$$

The $(L D, M C, M C)$ is defined by the following expression:

$$
\alpha_{1}<\frac{1}{2}, \alpha_{1}<\beta_{1}, \alpha_{2}>\frac{1}{2}, \beta_{2}>\frac{1}{2}, \alpha_{e f f}>\frac{1}{2}, \beta_{3}>\frac{1}{2}
$$

According to the law of conservation of flow in (1), we can obtain the following equation $J_{3}\left(\frac{1}{4}\right)=J_{2}\left(\frac{1}{4}\right)+J_{1}$. Hence, $J_{1} \approx 0$.

For phase $(M C, L D, M C)$, it should satisfy:

$$
\alpha_{1}>\frac{1}{2}, \beta_{2}>\frac{1}{2}, \alpha_{2}<\frac{1}{2}, \alpha_{2}<\beta_{2}, \alpha_{e f f}>\frac{1}{2}, \beta_{3}>\frac{1}{2}
$$

According to the law of conservation of flow in (1), we can obtain the following equation $J_{3}\left(\frac{1}{4}\right)=J_{1}\left(\frac{1}{4}\right)+J_{2}$. Hence, $J_{2} \approx 0$.

\section{Performance Evaluation}

In this chapter, firstly, we evaluate the performance of algorithm YELLOW in a small linear topology network. Afterwards, we use Monte Carlo simulation method to verify the accuracy of the approximate solution for the existence of conditions of each phase in YELLOW deduced by the TASEP model in the previous section. 


\subsection{The Performance of the YELLOW Algorithm}

To evaluate the performance of YELLOW, we carry out packet-level simulations using the NS-3 simulator [47], which is based on a discrete event. NS-3 abstracts the topologies, channels, network protocols, and some commonly network element devices used in real networks, and encapsulates them into common $\mathrm{C}++$ class objects. We deploy ICN packets upon the IP layer in the form of overlay, so that routing can be easily implemented through the default IP routing rather than complex routing-by-naming scheme like $\mathrm{CCN}$ [3]. We adopt the lookup-by-name mechanism [11,48] to help user find the named data chunk (NDC). Specifically, a hash table is set to record the mapping of the name of NDCs and their global addresses.

A robust and receiver-driven transport protocol, content-centric TCP (CCTCP) [20], is implemented to guarantee the transmission quality, which supports the mechanism of timeout request retransmission based on RTT and retransmission time-out (RTO) estimates. Besides, CCTCP limits the user's send window of NDC request in the AIMD model like TCP to avoid congestion. We deploy the algorithm YELLOW on the bottleneck link in a small linear topology network as shown in Figure 6. For a thorough performance comparison, we also implement two other classical AQM algorithms which are RED and BLUE. The ICN client application is implemented and installed in the nodes $\left(N_{1}, N_{2}, N_{3}\right)$ on the left side of the graph, which sends 10 requests for new NDC each seconds. The ICN server application is implemented and installed in the nodes $\left(N_{4}, N_{5}, N_{6}\right)$ on the right side and the routers $(\mathrm{A}, \mathrm{B}, \mathrm{C})$, which are responsible for responding the requests form client. As the most basic component in each end system, the ICN protocol stack ought to assemble received ICN packets to a chunk completely and sequentially.

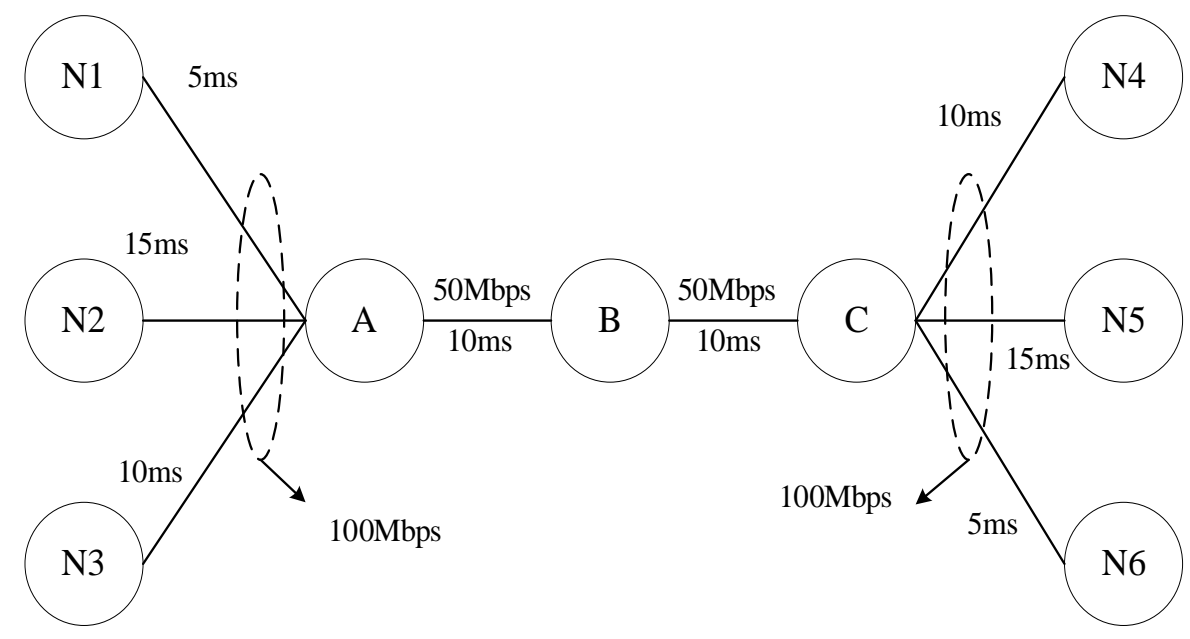

Figure 6. Simulation topology.

Chunk size is set to $256 \mathrm{~KB}$ to allow enough time for the AQM algorithms to make the client-end to adjust the request rate when congestion occurs. The workload extracted from catalog of $\mathrm{N}=1 \times 10^{5}$ content objects follows the Zipf distribution $(\alpha=0.8)$. The size of the cache store in router is 100 (chunks). The buffer of the queue and assembling memory of cache module accommodates 200 packets which, for 1500-byte packets, corresponds to a queuing delay of $0.35 \mathrm{~s}$. In order to ensure the same memory cost of YELLOW algorithm and comparison algorithms, the length of caching queue and forwarding queue are set to 100. Tables 3 and 4 shows the configurations used for RED and BLUE respectively, which is set according to the experience of previous studies [23,27]. 
Table 3. RED configuration.

\begin{tabular}{cc}
\hline Configuration & Value \\
\hline $\min _{t h}$ & $20 \%$ \\
$\max _{t h}$ & $80 \%$ \\
$\max _{p}$ & 1 \\
$w_{q}$ & 0.002 \\
\hline
\end{tabular}

Table 4. BLUE configuration.

\begin{tabular}{cc}
\hline Configuration & Value \\
\hline freeze_time & $50 \mathrm{~ms}$ \\
$\delta_{1}$ & 0.02 \\
$\delta_{2}$ & 0.002 \\
$\mathrm{~L}$ & 100 \\
\hline
\end{tabular}

In the following experiments, we find that even if YELLOW is set with different configurations, as long as it meets the parameter setting restrictions introduced in Section 3.3, the performance of YELLOW is not significantly different. For convenience of description, we set some configuration parameters to be the same as BLUE's, where freeze time $=50 \mathrm{~ms}$, $\delta_{1}=0.02, \delta_{2}=0.0025, \delta_{3}=0.001, L_{1}=80$ and $L_{2}=50$, mainly because the algorithm YELLOW is designed with reference to BLUE. The simulation program runs for $160 \mathrm{~s}$. The first $80 \mathrm{~s}$ are used to warm up the cache space of routers, and the remaining time is used to gather statistics. Specifically, the request rate is doubled at $20 \mathrm{~s}$ to simulate the more serious network congestion. At $60 \mathrm{~s}$, the request rate is reduced by one third to simulate the idle network. The sampling frequency of statistical data is 20 times per second, and then the average value is picked. To study the performance of algorithms under different ICN cache strategies, we also build two network scenarios adopting leave copy every (LCE) and leave copy down (LCD) [49], respectively. LCE caches chunks in every node along the transmission path. LCD improves the cache hit rate through storing the chunks only in the next hop of the previous cache node, which puts the popular contents close to users gradually. In order to fully understand the difference between YELLOW, RED and BLUE, we first observe the change of the queue length and marking probability under different loads and network scenarios as shown in Figures 7-9.

\section{LCE}

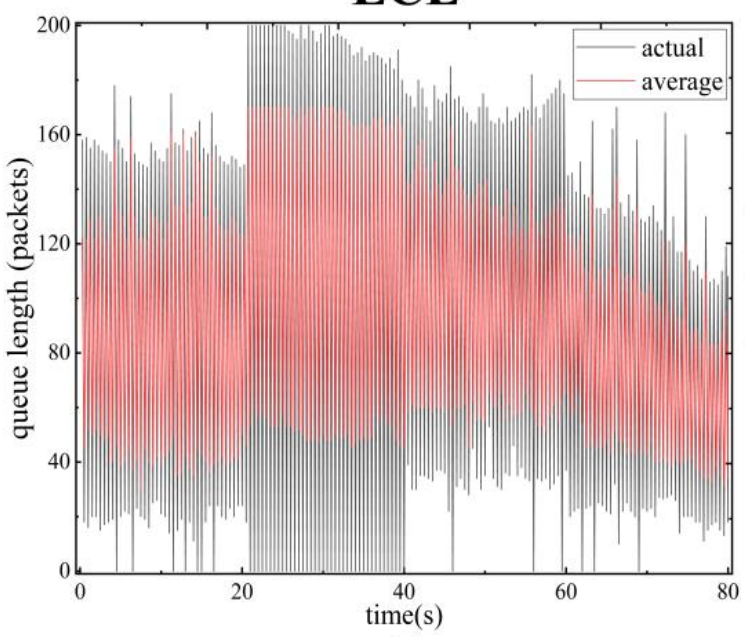

(a)

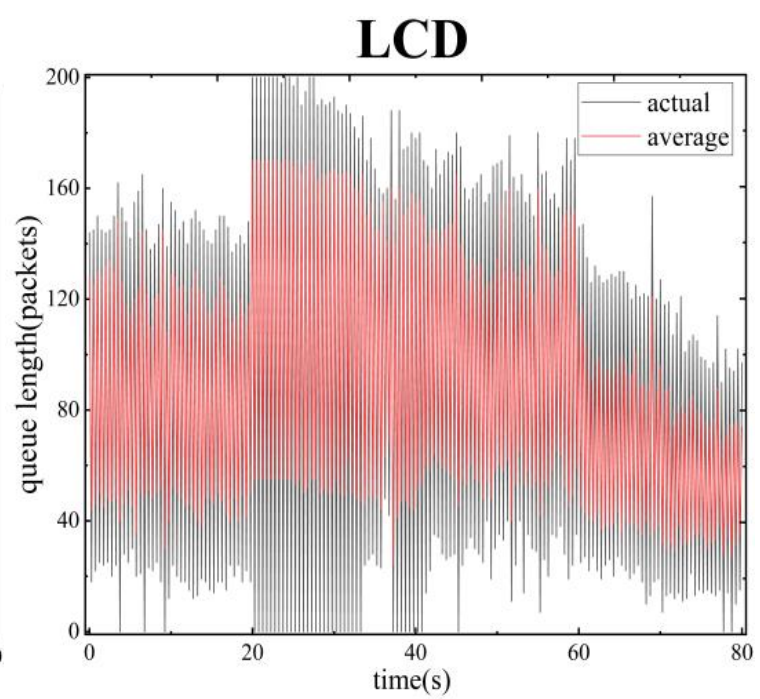

(b)

Figure 7. Cont. 


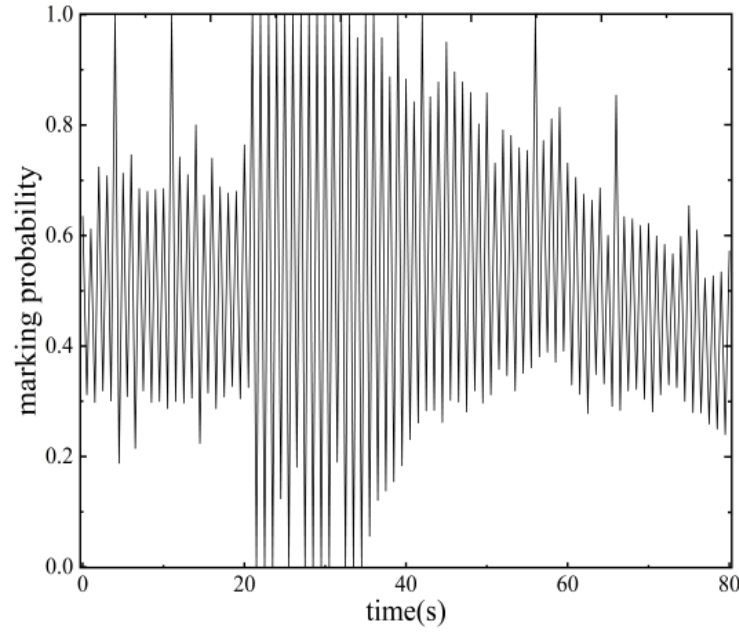

(c)

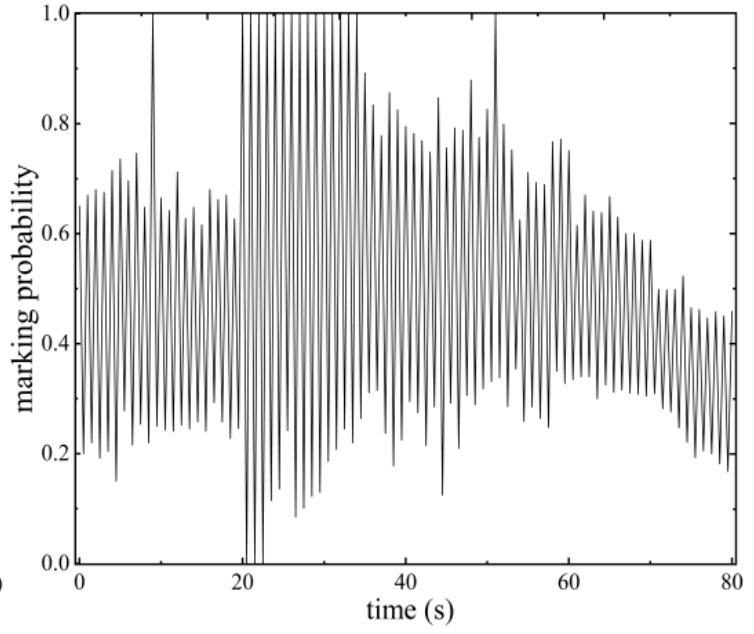

(d)

Figure 7. Comparison of queue length and marking probability under RED between leave copy every (LCE) and leave copy down (LCD). (a) the actual and average queue length of under LCE; (b) the actual and average queue length of under LCD; (c) the marking probability under LCE; (d) the marking probability under LCD.

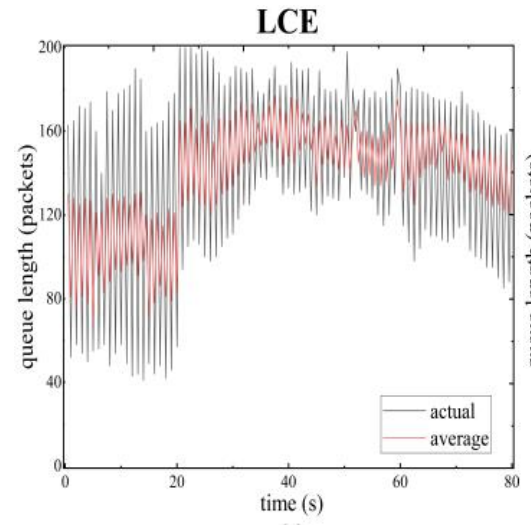

(a)

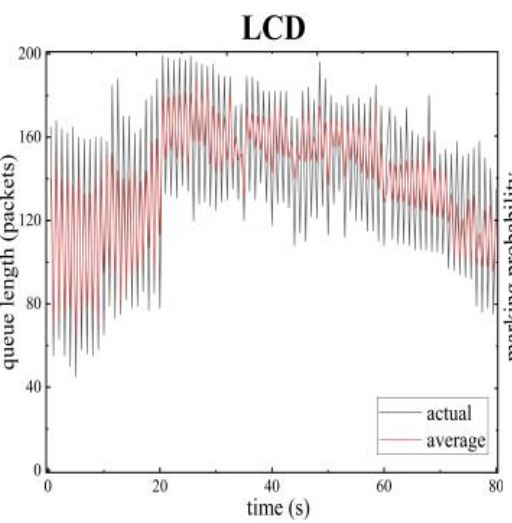

(b)

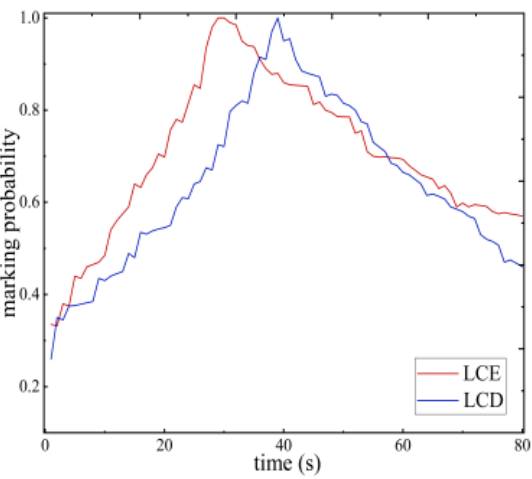

(c)

Figure 8. Comparison of queue length and marking probability under BLUE between LCE and LCD. (a) the actual and average queue length of under LCE; (b) the actual and average queue length of under LCD; (c) the marking probability under LCE and LCD.

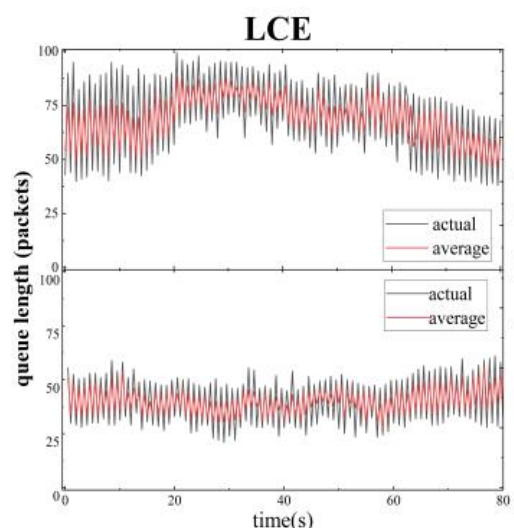

(a)

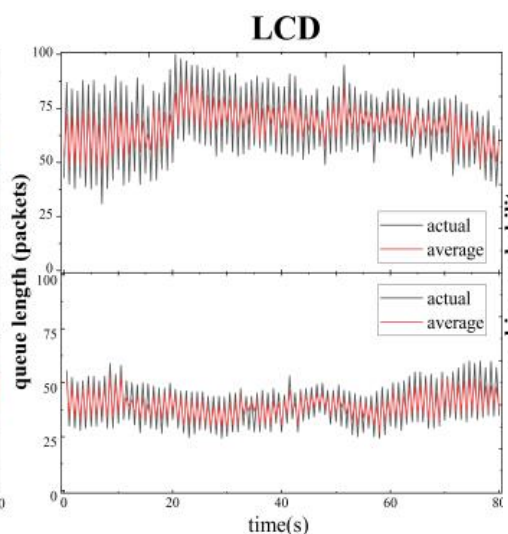

(b)

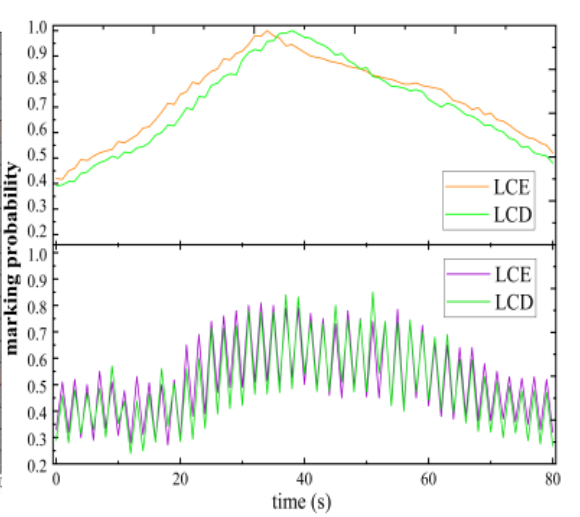

(c)

Figure 9. Comparison of queue length and marking probability under YELLOW between LCE and LCD. (a) the actual and average queue length of under LCE; (b) the actual and average queue length of under LCD; (c) the marking probability under LCE and LCD. 
The stability of queue length is an important index to judge the performance of an AQM algorithm. We observe that whether the network congestion is not obvious $(<20 \mathrm{~s})$ or the sudden traffic change (at $20 \mathrm{~s}$ or $60 \mathrm{~s}$ ), the queue length amplitude of RED is the largest, which means that the serious global synchronization is happening in the network. In the network scenarios with LCE, it is difficult for RED to keep the queue length between $\left[\min _{t h}, \max _{t h}\right]$ even when the traffic load is light (after $60 \mathrm{~s}$ ). Blue identifies whether congestion occurs through events of packet loss or link idle. BLUE performs better than RED because its marking probability is adjusted dynamically by increment. Although the memory utilization of the router has been improved, the queue length amplitude of BLUE is still large than YELLOW. Figure 9c shows that even if the network is busy, the oscillation amplitude of both forwarding queue (up) and caching service queue (down) in YELLOW can be maintained at a small level. Since the monitoring mechanism of bandwidth competition make a good prediction of the burst flow from the router, YELLOW also performs well in dealing with the burst traffic.

Marking probability is another important index to judge the algorithm performance, which allows us to explore the effectiveness of AQM in preventing global synchronization and in removing biases against burst sources. For RED, marking probability $p$ is equal to $p_{b} / 1-$ count $\times p_{b}$, where $p_{b}=\max _{p} \times\left(\left(Q_{a v g}-\min _{t h}\right) /\left(\max _{t h}-\min _{t h}\right)\right)$. We can find that $p_{b}$ is linear function of $Q_{a v g}$. The large shock of the average queue length will result in the marked probability changing significantly in a very short time (see Figure $7 c, d)$, which makes RED cannot relieve the synchronization phenomenon between content sources. By contrast, the marking probability of BLUE is relatively stable, which marks packets evenly even when the traffic is constantly fluctuating. The marking probability of YELLOW cache queue fluctuates while the marking probability of the forwarding queue can also keep a uniform value for a period of time. This is because YELLOW is self-adaptive, so it will adjust the send window of each cache service flow when router detects the change of forwarding throughput.

Moreover, the ICN protocol stack in router enables YELLOW to ensure the fairness of each burst response traffic easily. In general, YELLOW can perform better than BLUE/RED and obtain better robustness while eliminating the negative effects of global synchronization. Meanwhile, we find that different caching strategies also affect the performance of AQM algorithm. Compared with LCE, the LCD strategy improves cache-hit ratio and reduces the transmission traffic on the link greatly [49]. Therefore, no matter which AQM algorithm is adopted in the network with LCD, the fluctuation amplitude of the queue length is lower than that of LCE. Figures $7 d, 8 c$ and $9 c$ prove that the mark probability of AQM changes more smoothly in the network with LCD.

In order to better compare the performance of the three algorithms, Tables 5 and 6 list the bottleneck link utilization, throughput, download latency, average packet loss rate and the mean square error of queue length of the three algorithms under different network loads. We find that algorithm YELLOW can achieve the highest bottleneck link utilization and router throughput. Since low packet loss rate will increase the queuing delay, YELLOW with the lowest packet loss rate has the highest content download delay, followed by BLUE and RED. In terms of packet loss rate, YELLOW is also the best, Comparing the mean square error of queue length of three algorithms, we can obtain the same conclusion as the previous analysis about queue stability.

Table 5. Measurement data for light load network in time interval [0, 20].

\begin{tabular}{|c|c|c|c|c|}
\hline & RED & BLUE & \multicolumn{2}{|c|}{ YELLOW } \\
\hline bottleneck link utilization & $91.18 \%$ & $91.46 \%$ & \multicolumn{2}{|c|}{$91.75 \%$} \\
\hline Throughput (Mbps) & 48.13 & 48.52 & \multicolumn{2}{|c|}{48.75} \\
\hline average packet loss rate & $13.34 \%$ & $12.29 \%$ & \multicolumn{2}{|c|}{$11.45 \%$} \\
\hline Download latency (ms) & 148.73 & 160.82 & \multicolumn{2}{|c|}{169.1} \\
\hline \multirow{2}{*}{ mean square error of queue length } & \multirow{2}{*}{43.71} & \multirow{2}{*}{36.73} & forward & 17.48 \\
\hline & & & cache & 13.12 \\
\hline
\end{tabular}


Table 6. Measurement data for heavy load network in time interval [20, 40].

\begin{tabular}{|c|c|c|c|c|}
\hline & RED & BLUE & \multicolumn{2}{|c|}{ YELLOW } \\
\hline bottleneck link utilization & $94.08 \%$ & $94.18 \%$ & \multicolumn{2}{|c|}{$94.32 \%$} \\
\hline Throughput (Mbps) & 48.12 & 48.57 & \multicolumn{2}{|c|}{48.77} \\
\hline average packet loss rate & $21.78 \%$ & $19.48 \%$ & \multicolumn{2}{|c|}{$18.51 \%$} \\
\hline Download latency (ms) & 155.81 & 163.34 & \multicolumn{2}{|c|}{172.6} \\
\hline \multirow{2}{*}{ mean square error of queue length } & \multirow{2}{*}{50.05} & \multirow{2}{*}{42.86} & forward & 19.64 \\
\hline & & & cache & 14.33 \\
\hline
\end{tabular}

\subsection{Verifying the Accuracy of the Model of YELLOW through Monte Carlo Simulation}

We build a simulation system that is same as the model shown in Figure 5, which contains three queues (queue 1, queue 2 and queue 3) by the Monte Carlo method. queue 1 simulates buffer situation of forwarding traffic that comes from the external network while queue 2 simulates the situation of the cache traffic generated internally. The site scope of queue $1 /$ queue $2 /$ queue 3 with capacity $L$ ranges from $1 / \mathrm{L}+1 / 2 \mathrm{~L}+1$ to $\mathrm{L} / 2 \mathrm{~L} / 3 \mathrm{~L}$. In each Monte Carlo step (MCS), the system employing the random update mechanism updates $3 \mathrm{~L}$ times according to the following rules:

1. Selecting a site $i(1 \leq i \leq 3 L)$ randomly;

2. If $i=1 /(L+1)$, it means that the first site of queue 1 /queue 2 is selected. If there is no particle in this site, a new particle will be injected to system with probability $\alpha_{1} / \alpha_{2}$.

3. If $i=3 L$, it means that the output site of queue 3 is selected. A particle locating in this site will leaves the system with probability $\beta_{3}$ when the site is occupied

4. If the selected site is occupied by particle and the adjacent site on the right is empty, the particle will move to the adjacent site.

5. In addition to the above cases, we need to add some rules of YELLOW algorithm.

6. When the number of particles whether in queue 1 or queue 2 exceeds the threshold ( $\frac{L}{2}$ in this experiment), $\alpha_{2}$ will be decreased by a fixed value $\delta_{1}$ firstly. $\alpha_{1}$ will be reduced by $\delta_{2}$ only if the queue occupancy of queue 1 exceeds $50 \%$. Noted that the value of $\delta_{1}$ is setting more than one order of magnitude larger than $\delta_{2}$.

7. If $\alpha_{1}+\alpha_{2}>\beta_{3}, \alpha_{2}$ will also be decreased by $\delta_{1}$.

8. System increase the value of $\alpha_{1}$ and $\alpha_{2}$ by $\delta_{3}$ when the stationary phase (LD,LD,LD) exists for a period of time.

To simplify the parameter of the existence condition of the stationary phase, we consider indicating $\alpha_{2}$ by $n \alpha_{1}(0<n \leq 1)$. Therefore, Equations (4), (6) and (18) can be rewritten by $(L D, L D, L D)$ :

$$
\begin{gathered}
\alpha_{1}<\frac{n+1-\sqrt{2 n}}{2\left(1+n^{2}\right)}, \beta_{3}>\frac{1}{2} \\
\alpha_{1}<\frac{1+n-\sqrt{(1+n)^{2}-4\left(1+n^{2}\right) \beta(1-\beta)}}{2\left(1+n^{2}\right)}, \beta_{3}<\frac{1}{2}
\end{gathered}
$$

$(L D, L D, M C)$ :

$$
\alpha_{1}<\frac{1+n-\sqrt{(1+n)^{2}-4\left(1+n^{2}\right) \beta(1-\beta)}}{2\left(1+n^{2}\right)}, \beta_{3}>\frac{1}{2}
$$

$(H D, L D, M C)$ :

$$
\frac{n+1-\sqrt{2 n}}{2\left(1+n^{2}\right)}<\alpha_{1}<\frac{2-\sqrt{2}}{4 n}, \beta_{3}>\frac{1}{2}
$$

Figure 10 presents the phase diagram of model system on $\alpha_{1}-\beta_{3}$ axis when $n$ is fixed at 0.5 . Points and lines correspond to the results of simulation and analysis respectively. We find that there are symbiotic stationary phases in some phases. For example, there are unusual $(L D, L D, M C)$ in $(M C, L D, M C),(L D, H D, M C)$ in $(L D, M C, M C),(L D, L D, M C)$ 
in $(H D, L D, M C)$. The symbiotic phase exists because the system is simulating the YELLOW algorithm to relieve congestion so that probability $\alpha_{1}, \alpha_{2}$ will change in a small range over a period of time. However, due to the phase transition occurring in the time domain, the shock can not be seen in this coordinate system. The reason why there is no stationary coexistence phase in $(L D, M C, M C)$ is that the marking probability of queue 2 changes dramatically, which makes jumping phases only exist in a very short time. By contrast, the marking probability of queue 1 changes slowly and uniformly making it easy to stabilize for jumping phases (see experiment Figure 9c).

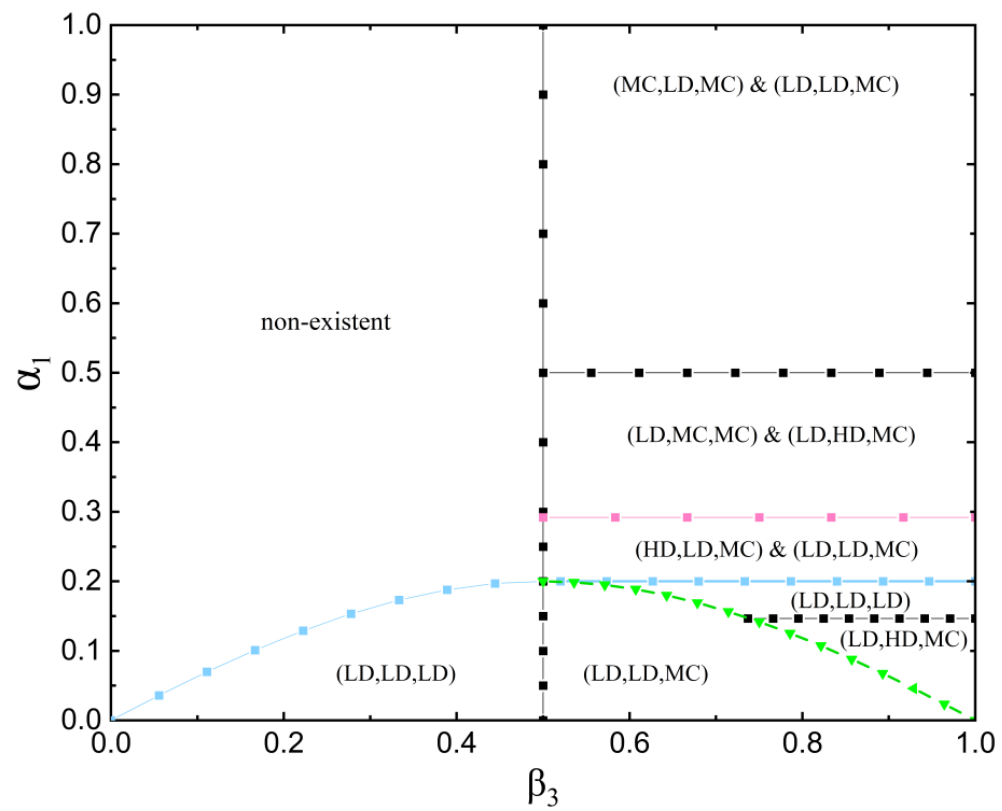

Figure 10. System phase diagram when $n$ is fixed at 0.5 .

Besides, as shown in Figure 11, with the decrease (increase) of $n$, the areas occupied by $(L D, L D, M C),(L D, L D, L D)$ and $(H D, L D, M C) \&(L D, L D, M C)$ gradually expands (shrinks). ( $L D, M C, M C) \&(L D, H D, M C)$ is the opposite. When the value of $\mathrm{n}$ is equal to 1 , the phase $(H D, L D, M C) \&(L D, L D, M C)$ disappears.

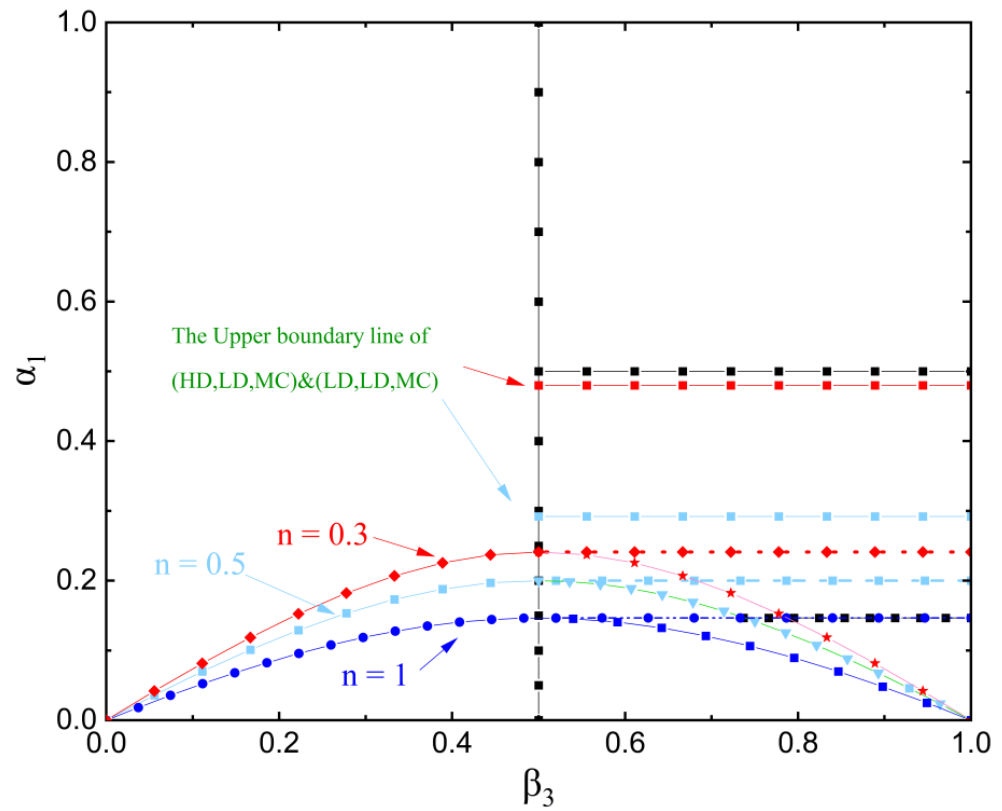

Figure 11. System phase diagram with variable n. 
Figure 12 presents the particle density distribution of six stationary phase running under specific parameters. The coordinate-axis $X$ is the sequential site in the queue $1 / 2 / 3$. Points and lines correspond to the results of simulation and analysis respectively. The simulation results are in good agreement with the analytical results. For the LD phase in the system, the density distribution is flat except near the queue boundary. Due to the adaptive adjustment of the YELLOW algorithm, the density distribution of queue 3 can converge to a fixed value around 0.5 regardless of the existence of shock at the queue boundary. Because the yellow algorithm reduces the packet loss rate of queue 1 in order to ensure the forwarding bandwidth, even if both queue 2 and queue 1 are in the LD phase at the same time, the particle density of queue 1 is significantly higher than that of queue 2. The shock appears at the head and tail of queue 3 at the same time, which is caused by the oscillation of the YELLOW algorithm in the time domain. Due to the coexistence phase existing in YELLOW modeling by TASEP, it is difficult to analyze the particle density distribution on each phase boundary by traditional domain-wall theory [50]. Therefore, we will not carry out the experimental analysis here, and will continue to study in the future.
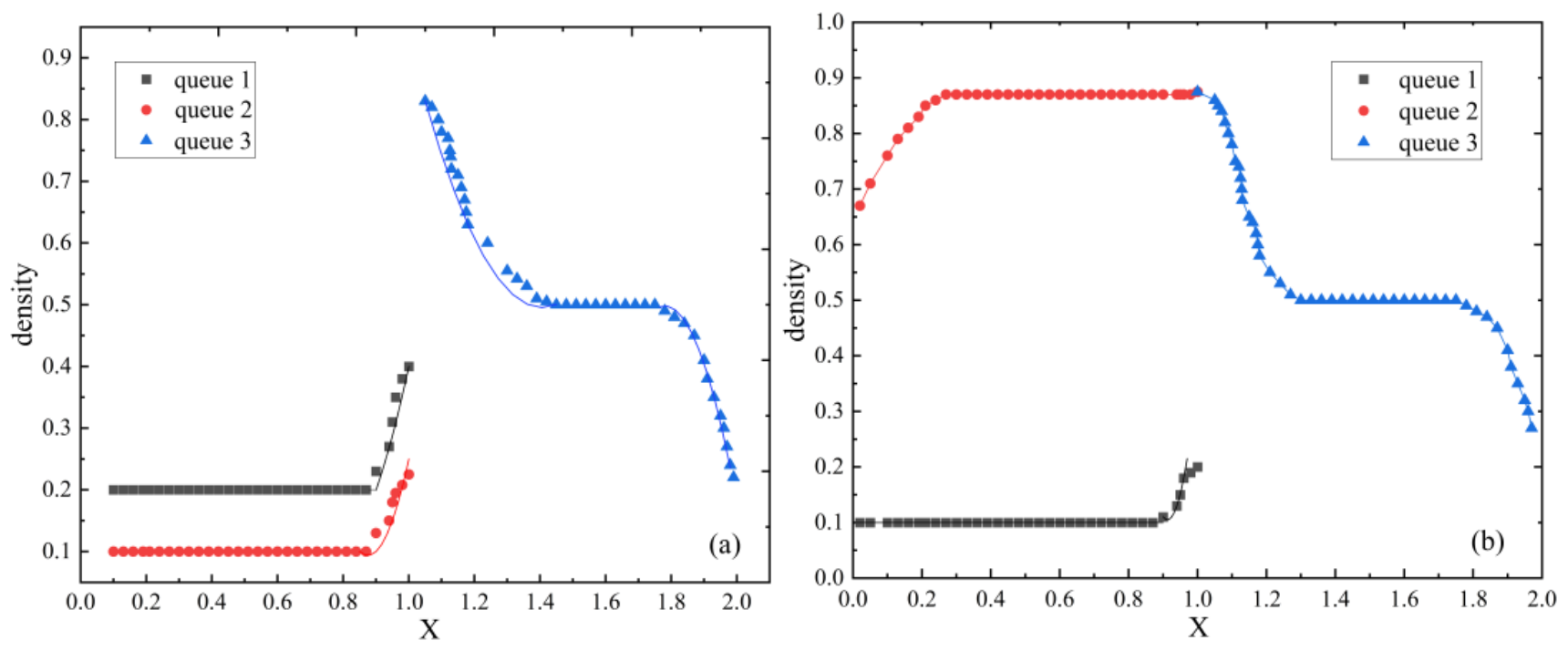

Figure 12. Cont. 

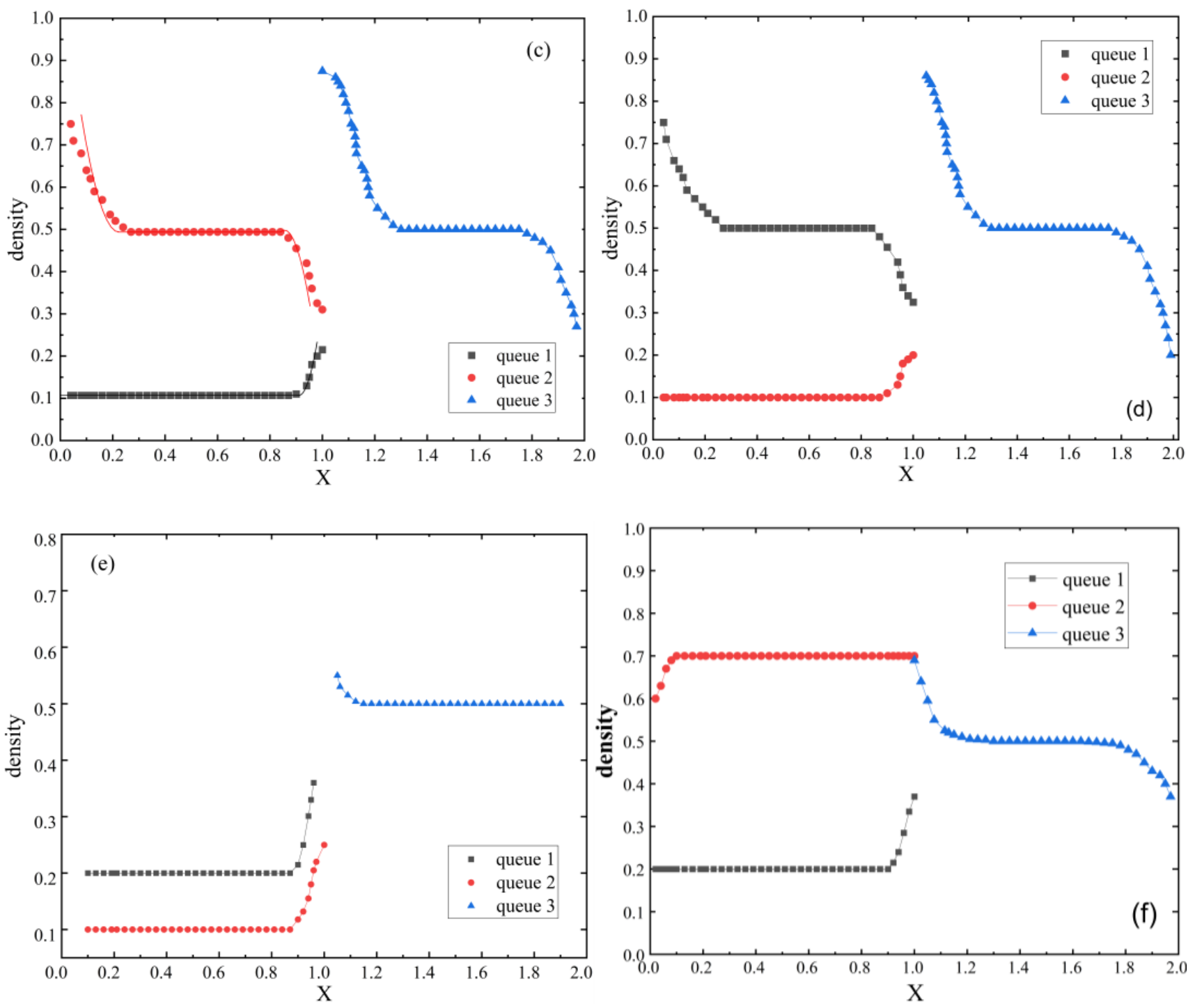

Figure 12. (a) $(L D, L D, M C), \alpha_{1}=0.2, \alpha_{2}=0.1, \beta=0.8$; (b) $(L D, H D, M C), \alpha_{1}=0.5, \alpha_{2}=0.1, \beta=0.8$; (c) $(L D, M C, M C), \alpha_{1}=0.1, \alpha_{2}=0.55, \beta=0.8 ;(\mathbf{d})(M C, L D, M C), \alpha_{1}=0.55, \alpha_{2}=0.1, \beta=0.8 ;(\mathbf{e})(L D, L D, L D)$, $\alpha_{1}=0.2, \alpha_{2}=0.1, \beta=0.4$; (f) $(H D, L D, M C), \alpha_{1}=0.25, \alpha_{2}=0.5, \beta=0.7$.

\section{Discussion}

In this paper, we consider that one of the main factors for congestion and packet loss in ICN routers with an additional end system can be further summarized as the competition between the external forwarding traffic and the internal cache response traffic for limited bandwidth resources. We propose an adaptive active queue management algorithm with dual queues for ICN routers, which predicts and limits the throughput of the response flow of burst request while guaranteeing the throughput of forwarding flow. We prove that ensuring the priority of forwarding traffic when allocating the bandwidth can achieve better benefits mathematically through probability theory. In addition, when bandwidth competition is predicted, YELLOW will limit the rate of request packets to enter the cache module of the router through a token bucket algorithm. In order to study the effect of packet arrival rate on the performance of the YELLOW algorithm, we model YELLOW through the widely studied TASEP model, and give the approximate solution of each stationary phase and the particle density.

Compared with the other two classical AQM algorithms (RED and BLUE) in network scenarios with different ICN cache strategies and load, the simulation results showed that the queue of YELLOW can be stable at the expected value, and the significant gains of 
router with low packet loss rate, robustness and high throughput. We verify the accuracy of the module results of YELLOW through Monte Carlo approximation while we find an interesting phenomenon that there are some symbiotic phases appearing in the system phase diagram. This is because the input/output rate of TASEP queues are adjusted adaptively in time domain when the YELLOW algorithm detects congestion.

We hope that this paper can provide ideas for more research on optimizing the existing ICN transport protocol and congestion control. Moreover, we will continue to study how to analyze and deduce the approximate solution of the existence condition of the symbiotic phase more accurately. In the future, we are interested in further research on how to ensure the fairness of each burst response flow in the router, and how to analyze the particle density distribution on the phase boundary.

Author Contributions: Conceptualization, L.Z., H.N., and R.H.; methodology, L.Z., H.N.; software, L.Z.; writing—original draft preparation, L.Z.; writing—review and editing, L.Z., H.N., and R.H.; supervision, H.N.; project administration, R.H.; funding acquisition, H.N. All authors have read and agreed to the published version of the manuscript.

Funding: This work was funded by Strategic Leadership Project of Chinese Academy of Sciences: SEANET Technology Standardization Research System Development (Project No. XDC02070100).

Acknowledgments: We would like to express our gratitude to Hong Ni, Rui Han, Yuanhang Li for their meaningful support for this work.

Conflicts of Interest: The authors declare no conflict of interest.

\section{References}

1. Cisco Visual Networking Index: Forecast and Methodology: 2017 2022. Available online: https:/ /www.cisco.com/c/en/us / solutions/collateral/executive-perspectives/annual-internet-report/white-paper-c11-741490.html. (accessed on 9 March 2018).

2. Dannewitz, C.; Kutscher, D.; Ohlman, B.; Farrell, S.; Ahlgren, B.; Karl, H. Network of Information (NetInf)—An informationcentric networking architecture. Comput. Commun. 2013, 36, 721-735. [CrossRef]

3. Wang, G.Q.; Zheng, Q.; Ravindran, R. Leveraging ICN for Secure Content Distribution in IP Networks. In Proceedings of the 7th ACM International Conference on Multimedia System (MMSys), Klagenfurt am Wörthersee, Austria, 10-13 May 2016; pp. 765-767.

4. Jacobson, V.; Smetters, D.K.; Thornton, J.D.; Plass, M.F.; Briggs, N.H.; Braynard, R.L. Networking named content. In Proceedings of the 5th International Conference on Emerging Networking Experiments, Rome, Italy, 1-4 December 2009; pp. 1-12.

5. Zhang, L.; Alexander, A.; Jeffrey, B.; Jackson, V.; Claffy, K.C. Named data networking. ACM SIGCOMM Comput. Commun. Rev. 2014, 44, 66-73. [CrossRef]

6. Peng, G. CDN: Content distribution network. arXiv 2004, arXiv:preprint cs/0411069.

7. Hinden, R.M.; Deering, S.E. Internet Protocol, Version 6 (IPv6) Specification. Rtp Udp Esp Uncompressed. 1981. Available online: https: / / www.rfc-editor.org/pdfrfc/rfc2460.txt.pdf (accessed on 29 March 2021).

8. Thomas, Y.; Xylomenos, G.; Tsilopoulos, C.; Polyzos, G.C. Object-oriented packet caching for ICN. In Proceedings of the 2nd ACM International Conference on Information-Centric Networking, San Francisco, CA, USA, 30 September-2 October 2015; pp. 89-98.

9. Rossini, G.; Rossi, D.; Garetto, M.; Leonardi, E. Multi-terabyte and multi-Gbps information centric routers. In Proceedings of the IEEE Conference on Computer Communications(INFOCOM), Toronto, ON, Canada, 27 April-2 May 2014; pp. 181-189.

10. Ding, L.; Wang, J.L.; Sheng, Y.Q.; Wang, L.F. A Split Architecture Approach to Terabyte-Scale Caching in a Protocol-Oblivious Forwarding Switch. IEEE Trans. Netw. Serv. Manag. 2017, 14, 1171-1184. [CrossRef]

11. Zeng, L.; Ni, H.; Han, R. An Incrementally Deployable IP-Compatible-Information-Centric Networking Hierarchical Cache System. Appl. Sci. 2020, 10, 6228. [CrossRef]

12. Storage Performance Development Kit. Available online: http:/ / www.spdk.io/ (accessed on 17 June 2017).

13. Stefano, S.; Andrea, D.; Matteo, C.; Pomposini, M.; Blefari-Melazzi, N. Transport-layer issues in information centric networks. In Proceedings of the Second Edition of the ICN Workshop on Information-Centric Networking, Helsinki, Finland, 17 August 2012; pp. 19-24.

14. Chai, W.K.; Diliang, H.; Ioannis, P.; George, P. Cache "less for more" in information-centric networks. In Proceedings of the International Conference on Research in Networking, Berlin, Heidelberg, 21-25 May 2012; pp. $27-40$.

15. Yang, Q.; Deng, H.; Wang, L. A lightweight caching decision strategy based on node edge-degree for information centric networking. IEEE Access 2020, 99, 1. [CrossRef]

16. Ha, N.V.; Kumazoe, K.; Tsuru, M. Tcp network coding with enhanced retransmission for heavy and bursty loss. IEICE Trans. Commun. 2017, E100-B(2), 293-303. 
17. Kunniyur, S.; Srikant, R. End-to-end congestion control schemes: Utility functions, random losses and ECN marks. IEEE/ACM Trans. Netw. 2003, 11, 689-702. [CrossRef]

18. Carofiglio, G.; Gallo, M.; Muscariello, L. ICP: Design and evaluation of an Interest control protocol for content-centric networking. In Proceedings of the 2012 IEEE INFOCOM Workshops, Orlando, FL, USA, 25-30 March 2012; pp. 304-309.

19. Salsano, S.; Detti, A.; Cancellieri, M.; Pomposini, M.; Blefari-Melazzi, N. Receiver-driven interest control protocol for contentcentric networks. In Proceedings of the ACM SIGCOMM Workshop on Information Centric Networking (ICN), Toronto, ON, Canada, 12-15 August 2012.

20. Saino, L.; Cocora, C.; Pavlou, G. CCTCP: A scalable receiver-driven congestion control protocol for content centric networking. In Proceedings of the 2013 IEEE International Conference on Communications (ICC), Budapest, Hungary, 9-13 June 2013; pp. 3775-3780.

21. Rozhnova, N.; Fdida, S. An effective hop-by-hop interest shaping mechanism for ccn communications. In Proceedings of the 2012 IEEE INFOCOM Workshops, Orlando, FL, USA, 25-30 March 2012; pp. 322-327.

22. Park, H.; Jang, H.; Kwon, T. Popularity-based congestion control in named data networking. In Proceedings of the 2014 sixth international conference on ubiquitous and future networks (ICUFN), Xi'an, China, 12-14 August 2014; pp. $166-171$.

23. Floyd, S.; Jacobson, V. Random early detection gateways for congestion avoidance. IEEE/ACM Trans. Netw. 1993, 1, 397-413. [CrossRef]

24. Floyd, S.; Jacobson, V. Recommendation on Using the Gentle Variant of RED. Available online: www.icir.org/floyd/red/gentle. html (accessed on 10 September 2000).

25. Li, Z.H.; Liu, Y.; Jing, Y.W. Active queue management algorithm for TCP networks with integral backstepping and minimax. Int. J. Control Autom. Syst. 2019, 17, 1059-1066. [CrossRef]

26. Kim, J.H.; Yoon, H.; Yeom, I. Active queue management for flow fairness and stable queue length. IEEE Trans. Parallel Distrib. Syst. 2010, 22, 571-579. [CrossRef]

27. Feng, W.-C.; Shin, K.; Kandlur, D.; Saha, D. The BLUE active queue management algorithms. IEEE/ACM Trans. Netw. 2002, 10, 513-528. [CrossRef]

28. Misra, V.; Gong, W.B.; Towsley, D. Fluid-based analysis of a network of AQM routers supporting TCP flows with an application to RED. In Proceedings of the Conference on Applications, Technologies, Architectures, and Protocols for Computer Communication, Stockholm, Sweden, 28 August-1 September 2000; pp. 151-160.

29. Kunniyur, S.; Srikant, R. Analysis and design of an adaptive virtual queue (AVQ) algorithm for active queue management. ACM SIGCOMM Comput. Commun. Rev. 2001, 31, 123-134. [CrossRef]

30. Ünal, H.U.; Melchor-Aguilar, D.; Üstebay, D.; Niculescu, S.I.; Özbay, H. Comparison of PI controllers designed for the delay model of TCP/AQM networks. Comput. Commun. 2013, 36, 1225-1234. [CrossRef]

31. Wang, K.; Jing, Y.; Liu, Y.; Liu, X.; Dimirovski, G.M. Adaptive finite-time congestion controller design of TCP/AQM systems based on neural network and funnel control. Neural Comput. Appl. 2020, 32, 9471-9478. [CrossRef]

32. Bisoy, S.K.; Pattnaik, P.K. An AQM controller based on feed-forward neural networks for stable internet. Arab. J. Sci. Eng. 2018, 43, 3993-4004. [CrossRef]

33. Ye, Q.; ZhuangZhuang, W.; Li, X.; Rao, J. End-to-End Delay Modeling for Embedded VNF Chains in 5G Core Networks. Internet Things J. IEEE 2019, 6, 692-704. [CrossRef]

34. Di Mauro, M.; Liotta, A.; Longo, M.; Postiglione, F. Statistical Characterization of Containerized IP Multimedia Subsystem through Queueing Networks. In Proceedings of the 6th IEEE International Conference on Network Softwarization (NetSoft), Chongqing, China, 12-15 June 2020; pp. 377-402.

35. Fu, T.Z.J.; Ding, J.; Ma, R.T.B.; Winslett, M.; Yang, Y.; Zhang, Z. DRS: Auto-Scaling for Real-Time Stream Analytics. IEEE/ACM Trans. Netw. 2017, 99, 1-15. [CrossRef]

36. Fu, T.Z.; Ding, J.; Ma, R.T.; Winslett, M.; Yang, Y.; Zhang, Z. DRS: Dynamic Resource Scheduling for Real-Time Analytics over Fast Streams. In Proceedings of the IEEE International Conference on Distributed Computing Systems, Fortaleza, Brazil, 10-20 June 2015; pp. 411-420.

37. Vakilinia, S.; Elbiaze, H. Latency control of ICN enabled 5G networks. J. Netw. Syst. Manag. 2020, 28, 81-107. [CrossRef]

38. Wang, M.; Yue, M.; Wu, Z. WinCM: A Window based Congestion Control Mechanism for NDN. In Proceedings of the 2018 1st IEEE International Conference on Hot Information-Centric Networking (HotICN), Shenzhen, China, 15-17 August 2018; pp. 80-86.

39. Amadeo, M.; Molinaro, A.; Campolo, C.; Sifalakis, M.; Tschudin, C. Transport layer design for named data wireless networking. In Proceedings of the 2014 IEEE conference on computer communications workshops (INFOCOM WKSHPS), Toronto, ON, Canada, 27 April-2 May 2014; pp. 464-469.

40. Karami, A. Accpndn: Adaptive congestion control protocol in named data networking by learning capacities using optimized time-lagged feedforward neural network. J. Netw. Comput. Appl. 2015, 56, 1-18. [CrossRef]

41. Liu, Y.F.; Zeng, X.W.; Han, R.; Sun, P. Toward ICN Receiver-Driven Transmission Mechanism over WLAN: Implementation and Optimization. Int. J. Innov. Comput. Inf. Control 2021, Accepted.

42. Wolfram, S. Statistical mechanics of cellular automata. Rev. Mod. Phys. 1983, 55, 601. [CrossRef]

43. De, G.J.; Essler, F.H.L. Bethe ansatz solution of the asymmetric exclusion process with open boundaries. Phys. Rev. Lett. 2005, $95,240601$. 
44. Derrida, B. An exactly soluble non-equilibrium system: The asymmetric simple exclusion process. Phys. Rep. 1998, $301,65-83$. [CrossRef]

45. Wang, Y.Q.; Zhang, Z.H. Cluster mean-field dynamics in one-dimensional TASEP with inner interactions and Langmuir dynamics. Mod. Phys. Lett. B 2019, 33, 1950012. [CrossRef]

46. Cook, L.J.; Zia, R.K. Power Spectra of a Totally Asymmetric Simple Exclusion Process with Finite Resources. In Proceedings of the APS March Meeting Abstracts, Portland, Oregon, 15-19 March 2010; p. T13-004.

47. NS-3 Project. Available online: https:/ / www.nsnam.og/ (accessed on 3 June 2014).

48. Wang, J.L.; Cheng, G.; You, J.L.; Sun, P. SEANet:Architecture and Technologies of an On-site, Elastic, Autonomous Network. J. Netw. New Media 2020, 6, 1-8.

49. Laoutaris, N.; Che, H.; Stavrakakis, I. The LCD interconnection of LRU caches and its analysis. Perform. Eval. 2006, 63, 609-634. [CrossRef]

50. Cividini, J.; Hilhorst, H.J.; Appert-Rolland, C. Exact domain wall theory for deterministic TASEP with parallel update. J. Phys. A Math. Theor. 2014, 47, 222001. [CrossRef] 